\title{
The Kinematics and Kinetics of Pedestrians on a Laterally Swaying Footbridge
}

\author{
D Claff*, MS Williams, A Blakeborough \\ Department of Engineering Science, Oxford University, Parks Road, Oxford, OX1 3PJ
}

\begin{abstract}
Progress in understanding human-structure interaction (HSI) on footbridges has been hampered by the shortage of quality data collected in realistic environments. This paper reports a novel experiment conducted on a naturally-swaying $7 \mathrm{~m}$ footbridge of frequency $0.67 \mathrm{~Hz}$ and amplitudes up to $125 \mathrm{~mm}$. Subjects crossed the bridge while wearing infrared motion-tracking markers and pressure-sensing insoles. The bridge was fitted with bespoke force plates, allowing investigators to simultaneously record kinematic and kinetic reactions to the structure's motion, which was assumed simple harmonic. The bridge was naturally excited by the test subjects, who were allowed to walk at a comfortable self-chosen pace. The data show that the subjects adopted a fixed-in-space Centre of Mass (CoM) strategy but their Centres of Pressure (CoP) were highly correlated to the position of the bridge deck within its lateral oscillation cycle (henceforth 'bridge phase'), allowing for the prediction of wide and crossed steps. Ground forces generally correlated to CoM$\mathrm{CoP}$ separation, which reflected the phase of the bridge at the previous heel-strike. Instantaneous step width was correlated to the bridge phase and is proportional to the offset in the Medial-Lateral (M-L) ground force between consecutive steps. The Inverted Pendulum Model (IPM) was evaluated using the data, exhibiting a limited fit to the recorded ground forces. Finally, the pedestrian-induced work on the bridge and system stability boundaries are also calculated, revealing mechanisms for bridge instability to occur.
\end{abstract}

Keywords: gait, bridge excitation, human induced vibration, inverted pendulum model, kinematics

\section{Introduction}

Since the late 20th century long, slender, and elegant bridges have become more prevalent, often risking resonance at low frequencies of excitation. This has particularly been observed in footbridges, where crowds exert lateral forces in the range of 0.5-1.3 Hz. Since the Pont du Solferino [1] and London Millennium Bridge [2] incidents, many tests have been conducted in an attempt to predict human-structure interactions (HSI) with footbridges. The goals of these tests are to understand how human gait adapts to the motion of a (laterally) moving base and to predict the (lateral) ground forces that cause problematic bridge vibrations to occur.

The nature of these tests varies significantly, including computer modelling [3], deterministic [4-6] and stochastic modelling [7, 8], treadmill experimentation [9, 10], and actuated platform tests [11-13]. These studies have produced a similarly wide variety of results with varying degrees of agreement [14].

Unfortunately, most of these studies have limitations as well. Field studies are not only limited by the permissions that must be obtained, but by the constraints imposed by instrumentation as well-especially when attempting to track individual pedestrians in a group. Computer models require an immense number of

*Corresponding author. Tel: +44(0)1865-273102 E-mail: declaff@gmail.com 
simplifying assumptions from data that are inconsistent or incomplete. Limitations also exist in laboratory tests: treadmill walking is inherently artificial and both platform and treadmill tests tend to be artificially actuated. Recent experiments (e.g. $[15,16])$ have sought to provide an increasingly realistic HSI experience in the lab, but even these rely on artificial actuation and the use of virtual reality. There is yet opportunity for improvement.

On the simulation side of HSI studies, one simple and popular walking model has been the Inverted Pendulum Model (IPM). This crudely represents a walking person as a lumped mass supported by two alternating rigid stick supports [17-19]. Using comparisons with fixed-base walking data, Claff [20] demonstrated the limitations of this model, in particular its inability to describe some of the higher-frequency, subjectunique components of lateral walking forces. Carroll et al. [15] achieve similar results, showing that the IPM captures neither the heel-strike impulse nor the high-frequency components of mid-step. Nevertheless, the model continues to be widely used in human-structure interaction studies due to its ease of use and its ability to capture many of the key characteristics of dynamic forces due to walking.

This paper presents the data obtained when instrumented individuals crossed a freely-swinging $7 \mathrm{~m}$ footbridge. The participants excited the structure naturally over two minutes while their natural kinematic and kinetic reactions were recorded. The objectives of the study were:

- to improve qualitative understanding of how human gait adapts to lateral base motion using a more realistic set-up than in previous experiments;

- to examine these adaptations on a step-by-step basis rather than a long-duration time series;

- to quantify Centre of Mass (CoM) and foot motion, Centre of Pressure (CoP) trajectory, and ground forces due to walking on a swaying structure. These key parameters are essential for the refinement of HSI models, which depend on relating body movement to ground forces for individual pedestrians;

- to develop correlations between body kinematics and structural motion, which has the potential to enable human-structure systems to be mathematically described entirely in terms of structural motion;

- to evaluate the accuracy with which the Inverted Pendulum Model can predict medial-lateral (M-L) ground forces on a moving base, in comparison with the measured data; and

- to compute the pedestrian-induced work on the bridge and examine conditions of bridge lateral instability, which highlight the engineering implications of the observed kinematic and kinetic data.

\section{Methods}

The centrepiece of the testing was the laboratory footbridge in the Department of Engineering Science at Oxford University. The bridge deck measures $7 \mathrm{~m}$ long by $1.2 \mathrm{~m}$ wide (Figure 1 ). The deck is suspended from a stationary steel frame in each of the four corners of the bridge via a compound pendulum that allows the bridge to swing laterally (Figure 2). The maximum amplitude of bridge displacement is limited by the width of the stationary steel frame, approximately $150 \mathrm{~mm}$. During peak lateral oscillation the pendulum motion causes the deck to elevate by approximately $25 \mathrm{~mm}$. This proportion of vertical to lateral motion was deemed sufficiently representative of long-span bridges prone to pedestrian lateral excitation. In situ structures are likely to exhibit some vertical and torsional motion in addition to lateral sway (e.g. [21]), although the proportion of vertical to lateral displacements will naturally vary from structure to structure. Nonetheless, the coupling of such vertical and horizontal deck motion may influence an individual's gait, but the effects were ignored in this study and deserve future examination. 


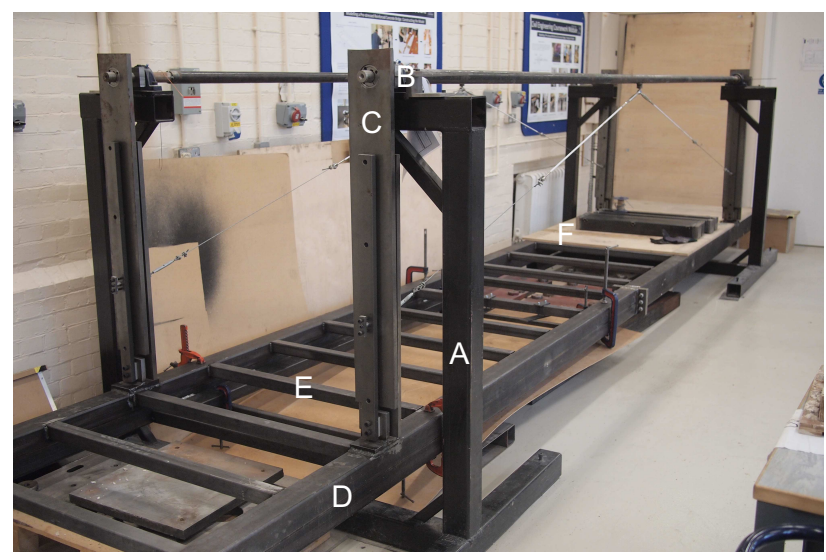

Figure 1: Configuration of the bridge before force plate installation. $(A)$ Structural support frame; $(B)$ Rotating bearing housing and circular handrail; $(C)$ Bearing pin assembly; $(D)$ Rectangular girders; $(E)$ Joists; and $(F)$ Plywood decking.
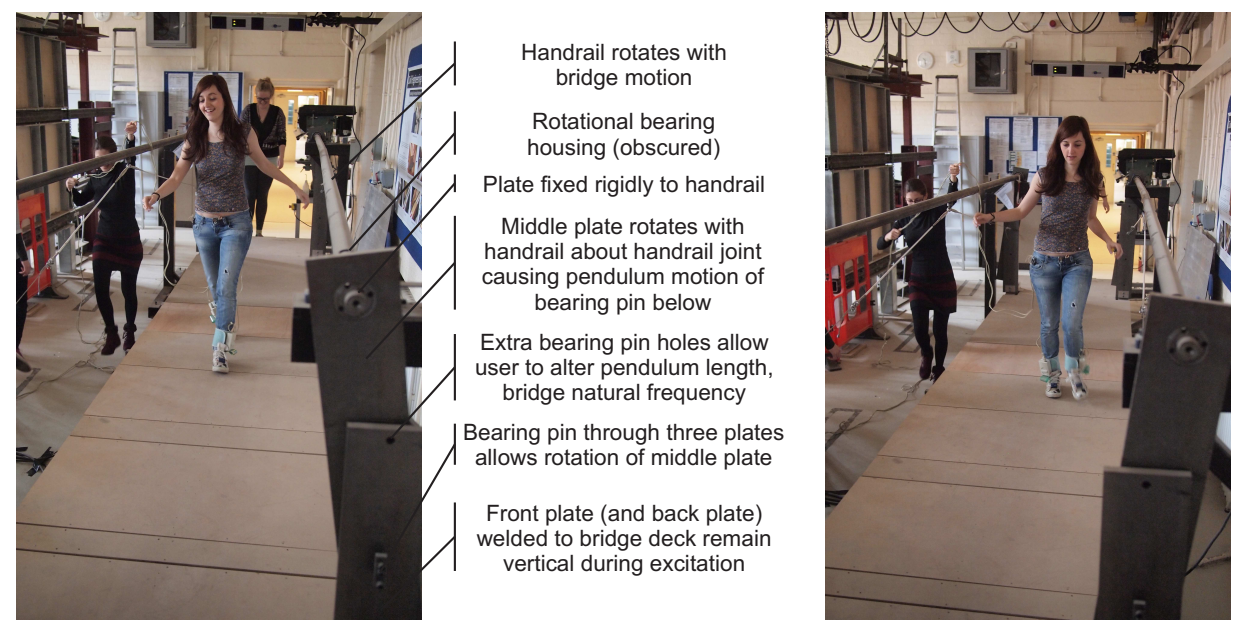

Figure 2: Motion of the bridge and bearing assembly during preliminary testing. Bridge swung to the subject's left (left photo) and to the subject's right (right photo), as determined by the position of the 'middle plate'.

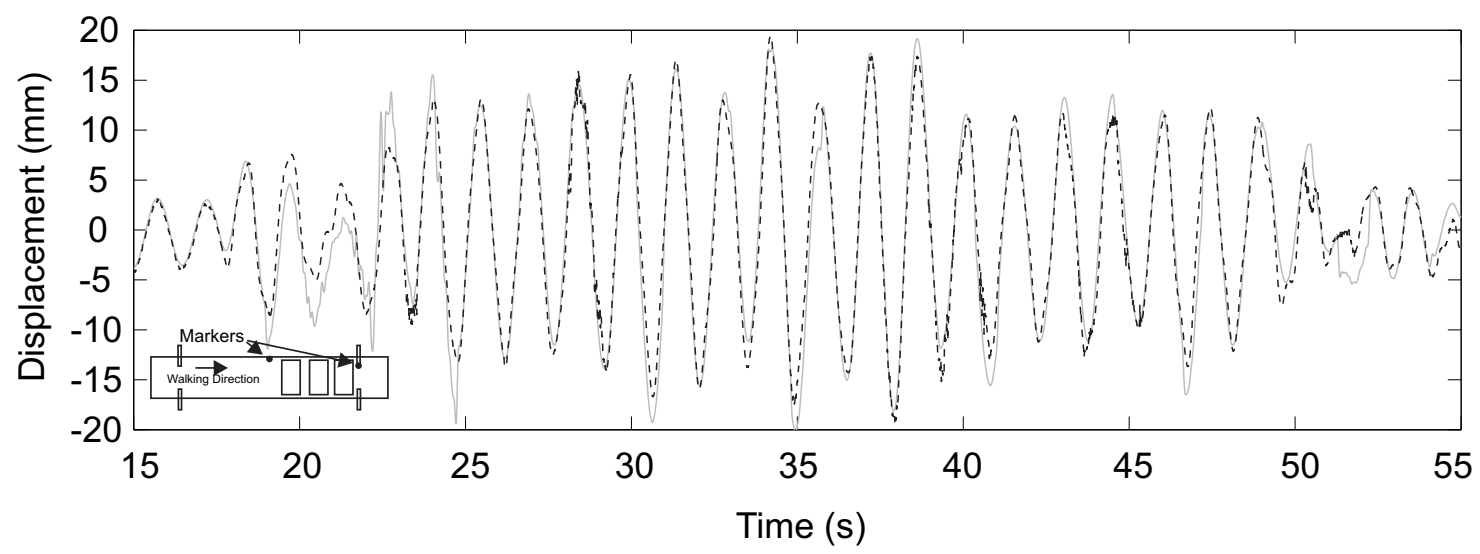

Figure 3: Lateral displacement of two bridge-mounted motion capture markers during one test, approximately 15 bridge crossings. Marker locations indicated in schematic. 


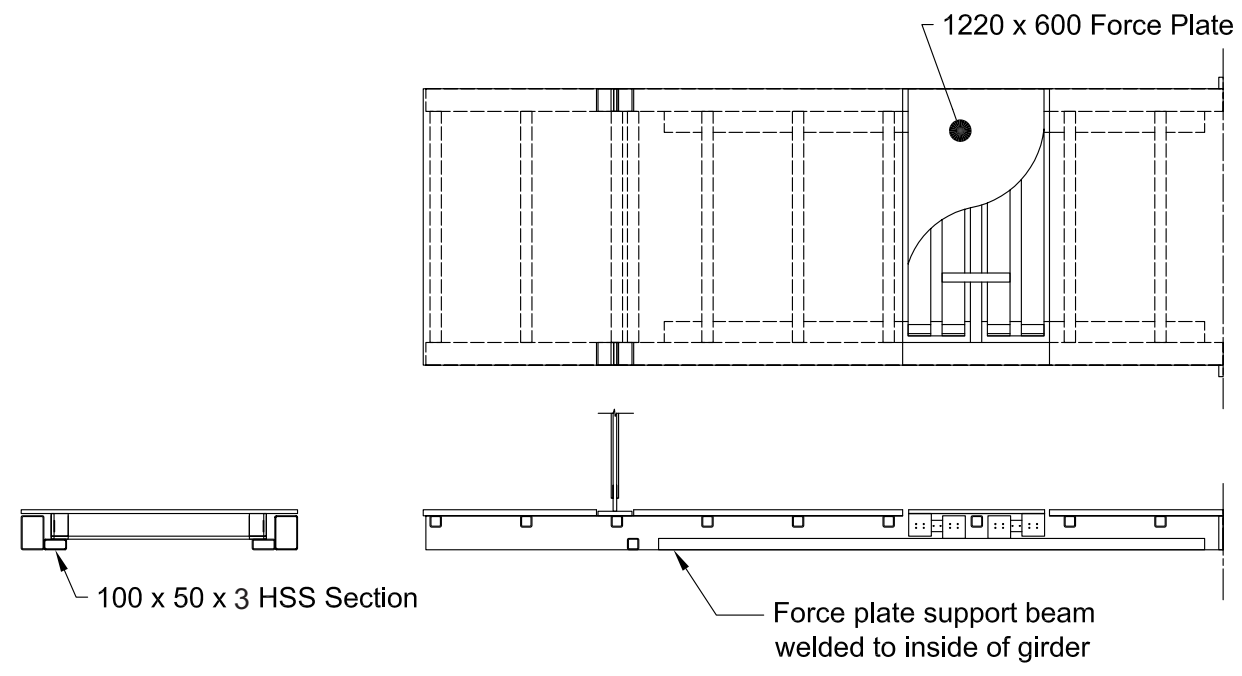

Figure 4: Half of bridge rig showing one force plate.

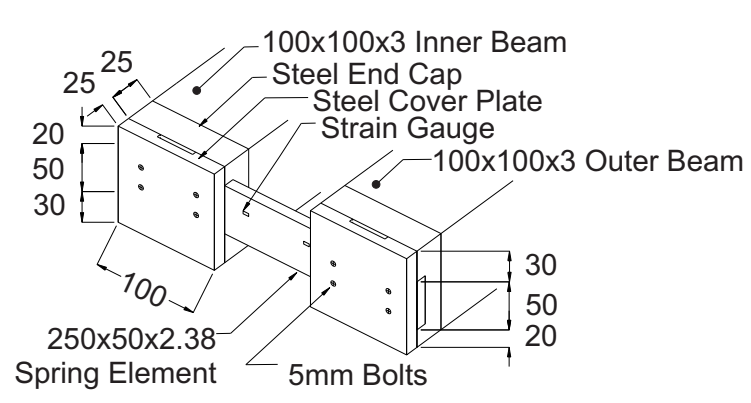

(a)

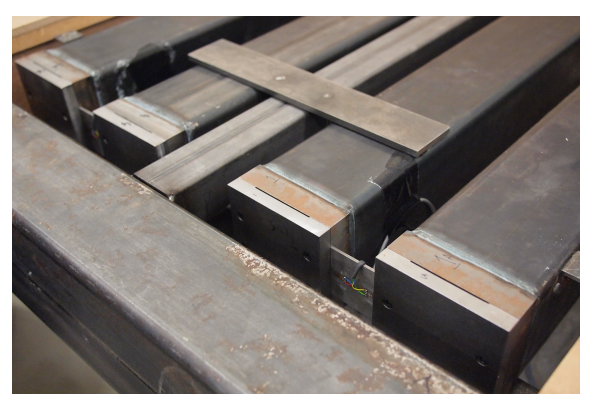

(b)

Figure 5: (a) Schematic of beam to spring element connections. All measurements in millimetres; not to scale. (b) Photograph shows the four beams that make up the force plate.

The superstructure of the bridge consists of two $150 \times 100 \times 4 \mathrm{~mm}$ steel rectangular hollow section girders joined by $50 \mathrm{~mm}$ square hollow section joists spaced at approximately $330 \mathrm{~mm}$ (See Figure 1). This is topped by a two-layer plywood deck of total thickness $28 \mathrm{~mm}$. The total superstructre mass is $1123 \mathrm{~kg}$. When unoccupied the bridge has a sway natural frequency of $0.67 \mathrm{~Hz}$ and is very lightly damped, with a damping ratio less than 0.001 . The bridge was assumed to sway in simple harmonic motion even though the imperfect rigidity of the structure and irregularity of the loading resulted in a slightly more complex dynamic system. Figure 3 shows a time history of lateral bridge displacement for $40 \mathrm{~s}$ of a test. The natural frequency is clearly visible throughout the time history. The power spectral density is dominated by the $0.67 \mathrm{~Hz}$ component. The deck had sufficient in-plane rigidity to move laterally as a rigid body, though limited flexibility in the support system meant that motion was not always purely translational. This is evident in the time history, where the mid-bridge and end-bridge markers were not always in perfect synchronisation. At approx. $20 \mathrm{~s}$ and $50 \mathrm{~s}$ the histories show that the markers deviated, indicative of $z$-axis rotation that was occasionally observed when a subject boarded the bridge. However, this rotation was minimal compared to the predominantly lateral swaying of the structure.

The superstructure contains three bespoke force plates designed to record lateral ground reaction forces while the bridge is in motion [22, 23]. Each force plate is $600 \mathrm{~mm}$ long in the direction of walking and spans 


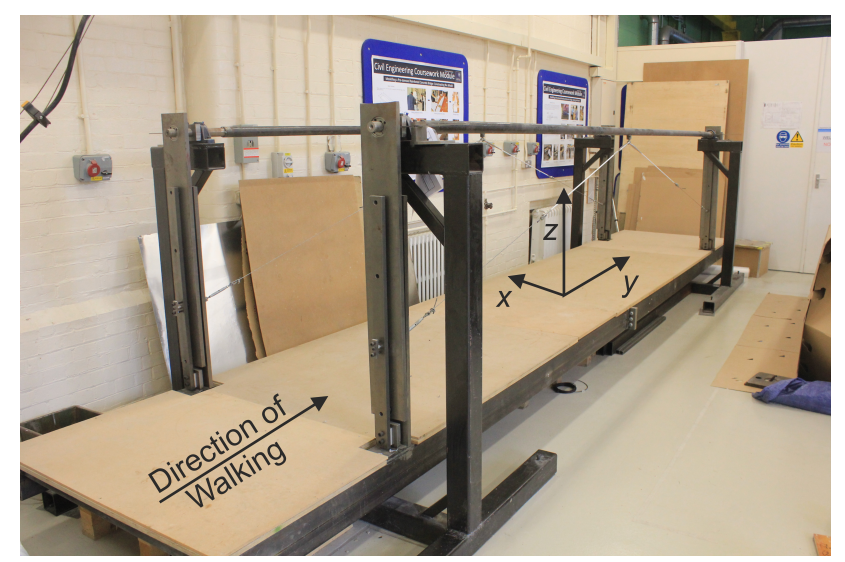

(a)

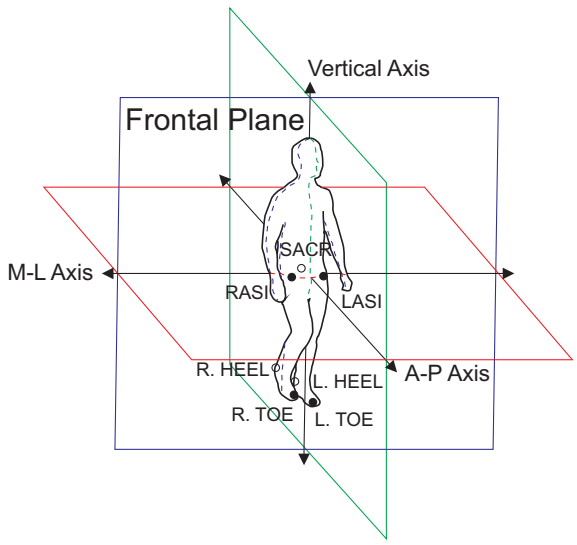

(b)

Figure 6: (a) A left-handed global coordinate system was used. (b) Schematic of marker positions. Filled dots show markers on front of body; empty dots show markers on backside of body.

the full deck width. Each force plate consists of four rigid steel beams in two pairs. Each pair is joined by two instrumented thin plate 'spring elements' that deflect laterally, recording a strain (Figures 4 and 5). In addition to measuring footfall forces, the plates developed inertial forces due to the lateral accelerations of the deck. These could be calculated from the deck motion, and the measurements adjusted accordingly; the accuracy of this process was verified using tests on the structure in the absence of walkers.

In addition to the force plates two other systems were used. A Codamotion CX1 motion tracking system was employed in order to record the motion of the CoM and feet of the participants as they crossed the bridge. The Codamotion sensors were mounted above each end of the bridge, creating a capture volume over the force plates approximately $3 \mathrm{~m}$ long. This established a global (i.e. absolute) coordinate system for which the origin was located at the centre of the at-rest bridge. For ease of post processing, the subjects' left is positive $x$-axis, forward is positive $y$, and up is positive $z$ : a left-handed coordinate system (Figure $6(\mathrm{a}))$.

Markers were mounted to each subjects' left and right anterior superior iliac spine (LASI, RASI), the sacrum (SACR), the heel of each shoe nearest to the spine of the Achilles tendon, and each big toenail (located as nearly as possible through the shoe) (Figure 6(b)). The marker set is based on the Oxford Gait Model, a standard set of markers developed by the Oxford Gait Laboratory (Nuffield Orthopaedic Centre, NHS Trust), which incorporates the Oxford Foot Model [24]. In addition, a marker was mounted to the bridge at mid-span and at the end support. The positions of the markers were recorded by Codamotion

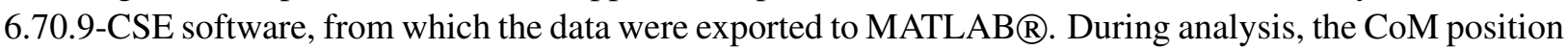
was estimated by averaging the LASI and RASI markers [23].

Finally a Tekscan F-scan pressure-tracking insole system was used to record the CoP of the participants. An insole was inserted into each participant's shoes and calibrated. Each insole consists of a grid of pressure-sensing nodes spaced $5.08 \mathrm{~mm}$ apart. The location of the big toe - and therefore the motion tracking marker - on the insole was estimated by asking each participant to press down with their shoes. The data were recorded in Tekscan's Foot Research 6.34 software, from which the local coordinates of the

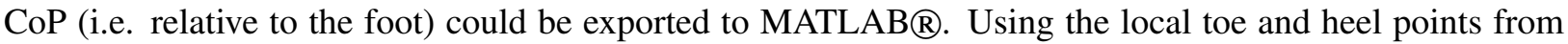
Tekscan along with the toe and heel CODA markers, the CoP coordinates from Tekscan were translated to the CODA (i.e. global) coordinate system using coordinate transformation matrices.

Like any set-up, this programme exhibited certain limitations. While the width of the structure did not 
Table 1: Sample Population Statistics.

\begin{tabular}{lccc}
\hline & Women & Men & Total \\
\cline { 2 - 4 } Number of People & 11 & 9 & 20 \\
Number of Recorded Trials & 312 & 253 & 566 \\
Three-Step Trials & 67 & 38 & 105 (315 steps) \\
& & & \\
Mass (kg) & $63.5 \pm 8.03$ & $80.1 \pm 21.06$ & $71.0 \pm 17.10$ \\
Height (m) & $1.70 \pm 0.0749$ & $1.81 \pm 0.0726$ & $1.75 \pm 0.0910$ \\
Age (years) & $25.8 \pm 2.32$ & $26.1 \pm 1.54$ & $26.0 \pm 1.96$ \\
\hline
\end{tabular}

appear to limit subject movement (only one individual out of 20 touched the handrails across the entire programme), entering and exiting the oscillating structure provided a transient impulse to both subject and structure. The subjects readily acclimated to the task, however, as if applying the familiarity of boarding an escalator. Moreover, the bridge was long enough to allow walkers to overcome this transient effect early in the transit and the force plates were positioned in the second half of the deck to minimise the influence of this effect. Another limitation of this set-up was the use of force plates, which requires the subject to have a certain stride length. The size and spacing of the plates was chosen to maximise clean strikes, but only 18.5\% (i.e. 105 out of 566) of bridge crossings landed three consecutive clean strikes.

Additionally, the CODA and Tekscan systems exhibited typical limitations for these systems. The former was prone to occlusions when the markers were obscured. Cubic fitting was employed to fill the occlusions. The Tekscan system was prone to aberrations due to creases in the in-soles, artefacts from malfunctioning nodes, and shoe-induced pressure (e.g. pressure occurring without weight on the foot), although these were minimised during subject-wise calibration. Finally, the transformation of Tekscan coordinates to global coordinates was limited by (1) the accuracy of mapping the CODA markers to the in-soles and (2) the possibility of the in-sole moving within the shoe relative to the CODA markers. As a result, errors to the CoP trajectory could be up to approximately $20 \mathrm{~mm}$ laterally.

Each participant recorded approximately four procedurally identical walking tests of two minutes each. Between each test occurred a resting period of approximately three minutes during which time the previous data was saved and the instrumentation reset. Participants used this time to catch their breath and the sway of the bridge was allowed to subside and/or stop.

During a test, the instrumented participant crossed the bridge repeatedly as many times as possible. Each crossing is henceforth defined as a trial; the mode number of trials that were completed during a test was six. If during a trial the participant stepped perfectly on all three force plates, that qualified as a 'clean three-step trial'. For each trial, the participant stepped up onto the bridge, crossed it, stepped down from the bridge, then walked around the bridge back to the start. Two non-instrumented participants crossed the bridge in succession with the instrumented participant in order to keep the bridge swaying. Only one individual was on the bridge at a time so that the forces exerted by and imparted on the subject were purely in response to his or her own walking.

Early experiments at prescribed stepping frequencies showed that subjects could not walk naturally and adapt to the bridge motion while maintaining the required tempo. In all the experiments reported here, therefore, subjects were encouraged to walk at a pace that felt natural and comfortable for them. This often led to them changing speed during a traverse in order to maintain balance and comfort. The resulting range of step frequencies (i.e. consecutive heel-strikes) was $0.76-1.2 \mathrm{~Hz}$ with a mean of $0.94 \mathrm{~Hz}$ and standard deviation of $0.083 \mathrm{~Hz}$. Note however that within the 93 tests, the mean step-to-step pacing change whilst crossing the force plates was $7.2 \%$, indicating that the subjects readily changed their step duration to accommodate structural motion. 
Table 1 shows the population statistics from the tests. Twenty total participants completed the 93 tests, resulting in 566 trials. Of those, 105 trials were clean three-step trials. The data from these trials forms the basis of this paper.

Ethical approval for these tests was obtained from the Oxford University Central University Research Ethics Committee (CUREC).

\section{Results}

In this section, results are presented and analysed to gain insights into gait on a swaying surface and the resulting ground forces.

First are the key elements of walking strategy that enable a person to cross a swaying structure without falling. The first of these elements is foot placement strategy, and how it compares to fixed-base walking. The second component of the walking strategy is how the CoP varies during a single foot placement, and how this is affected by bridge sway amplitude. Third is the role of CoM motion in the walking strategy. The examination of CoM frontal plane motion in the presence of high-amplitude bridge motion shows how destabilising bridge motions are mitigated by the upper body.

The paper then focuses on estimation of walking forces. Analysis of the CoP trajectories and force plate data is used to show that CoP-CoM separation is the primary determinant of M-L ground forces. The data are then used to evaluate the IPM for estimating lateral ground forces. Finally, the energy input to the bridge due to walking (which can be thought of as a negative damping effect) is quantified.

\subsection{Foot Placement}

Many have stated that foot placement is a primary determinant of gait. Few, however, have examined how the body affects foot placement on a laterally oscillating surface. Typically foot placement is measured in terms of step width, defined as the difference between two like points on opposite feet at the moment of heel-strike. This simplistic definition presents two challenges. First is the problem that different parts of the left and right feet are in contact with the ground at heel-strike. This paper overcomes that problem by using the separation of the left and right CoPs rather than the separation of, for example, the left and right heels.

The second challenge, given that the CoP is located in different places under each foot, is to define what is actually meant by step width. Suppose that a participant has consecutive heel-strikes at $x\left(t_{1}\right)=x_{1}$ and $x\left(t_{2}\right)=x_{2}$ where $t$ is time and $x$ is the CoP position in the M-L direction. The net step width (NSW) is thus henceforth defined as the $\mathrm{x}$-direction distance (i.e. lateral separation) between the CoPs of consectuive heel-strikes:

$$
\delta_{N}=x_{2}-x_{1}
$$

In contrast, the instantaneous step width (ISW) is henceforth defined as the lateral separation between the $\mathrm{CoPs}$ at the instant of the second heel-strike: a snapshot in time. Since the CoP of the first foot has migrated due to both bridge and its normal trajectory across the foot, $x\left(t_{2}\right)=x_{1}^{\prime}$ is taken to be the location of the CoP in the first foot when the second heel-strike occurs. Thus,

$$
\delta_{I}=x_{2}-x_{1}^{\prime} .
$$

The form of $\delta_{N}$ and $\delta_{I}$ is similar. Both compare the position of the second heel-strike with a CoP position from the first foot. The NSW compares the two heel-strike locations (despite the passage of time between their sampling) while ISW compares the two CoPs at an instant in time (despite the passage of the first CoP away from the heel). Both are beneficial because the NSW compares the relative locations of 


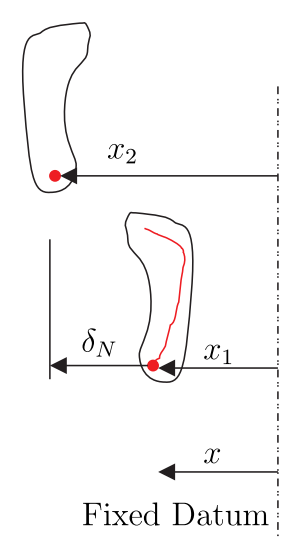

(A)

Net Step Width

(Fixed \& Moving Base)

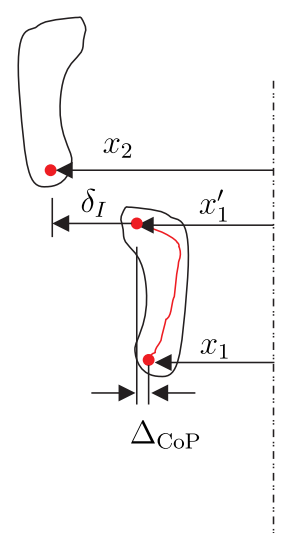

(B)

Instantaneous Step Width (Fixed Base)

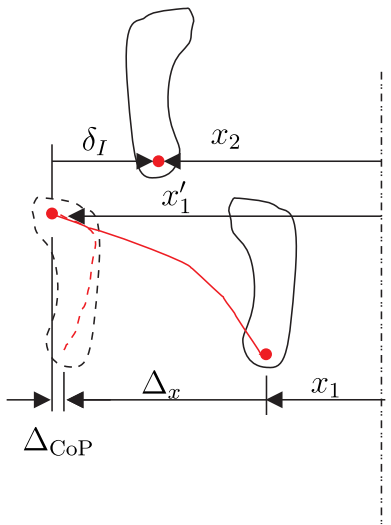

(C)

Instantaneous Step Width (Base Moving Left)

Figure 7: (A) Net Step Width $\left(\delta_{N}\right)$ versus (B) \& (C) Instantaneous Step Width $\left(\delta_{I}\right)$. Hypothetical CoP trajectory indicates displacement from heel at heel-strike to toe before toe-off. See text for discussion.

consecutive heel-strikes while the ISW provides a snapshot of the relative foot positions at the moment of the second heel-strike.

Note that $x_{1}^{\prime}$ can be written in terms of $x_{1}$ :

$$
x_{1}^{\prime}=x_{1}+\Delta_{\mathrm{CoP}}+\Delta_{x}
$$

where $\Delta_{x}$ is the lateral displacement of the bridge between consecutive heel-strike times and $\Delta_{\mathrm{CoP}}$ is the M-L displacement of the CoP within the foot due to walking. As a result, ISW can be written as

$$
\delta_{I}=x_{2}-\left(x_{1}+\Delta_{\mathrm{CoP}}+\Delta x\right)
$$

In Figure 7, three cases are depicted to help visualise the difference between NSW and ISW. Figure 7(a) shows the NSW. Since NSW only pertains to the relative position of consecutive heel-strikes, the same configuration applies to both fixed and moving base cases. Figure 7(b) shows the ISW case with no base motion. Here the CoP trajectory is included because ISW compares the second heel-strike position to the first foot $\mathrm{CoP}$ at that instant. One can clearly see the relationship described in Equation 2. Finally 7(c) is the case where base motion is to the left. Here (and keeping in mind that $x$ is positive leftward) Equation 2 causes ISW to be negative because $x_{2}$ is less than $x_{1}^{\prime}$. As a general rule of thumb, for large $\Delta_{x}$, it and $\delta_{I}$ will almost always be opposite signs.

Studies in the literature that refer to 'step width' most nearly discuss ISW. They compare the feet during double stance and investigate wide, normal, and crossed footsteps. However, these studies use the position of a motion tracking marker such as the heel, metatarsals, or an average rather than the actual CoP, so their analyses are not quite the same as that of the present study.

A crossing by one participant is shown in Figure 8. Each panel depicts the global position of a heelstrike and the toes just before toe-off. In Figure 8(a) the bridge is at its maximum leftward extent at HS1, so as the bridge moves rightward, the stance foot is carried with it. Figure 8(b) shows that TO1 is significantly to the right of where its heel-strike was located. In fact, it causes HS2 to be in line with or slightly crossed over TO1. In this configuration, the ISW is zero or slightly positive, but since HS2 is still to the right of HS1, NSW is negative. 


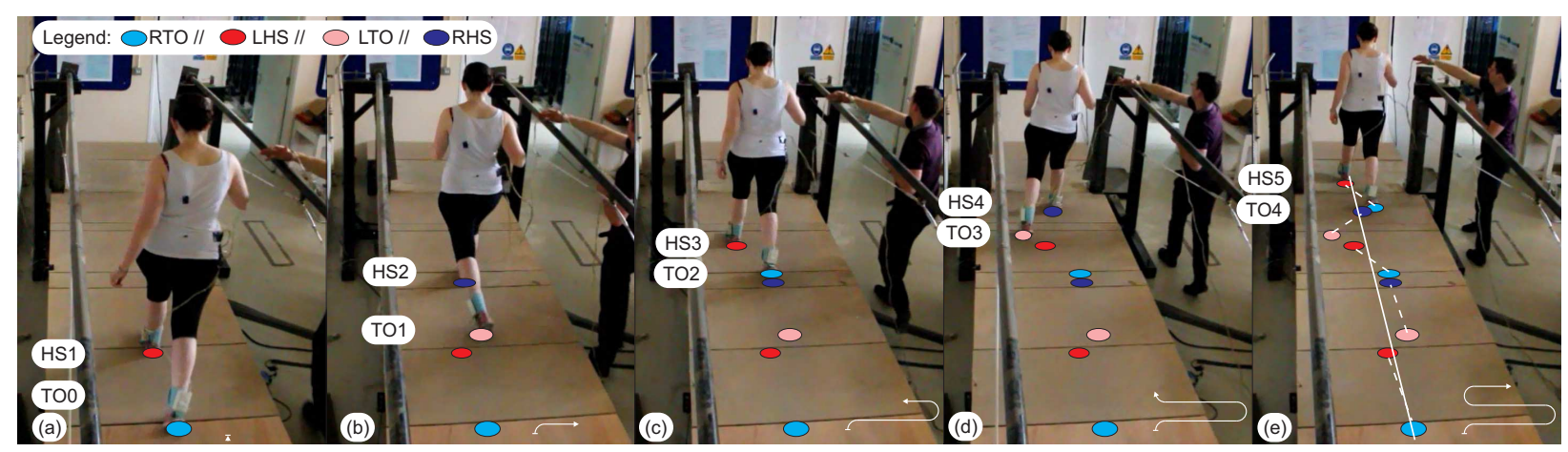

Figure 8: Sequence showing a participant crossing the bridge. Global position of heel-strikes (HS) and toe-offs (TO) indicated with dots. Cumulative bridge transit shown by arrow in each panel. See text for discussion.

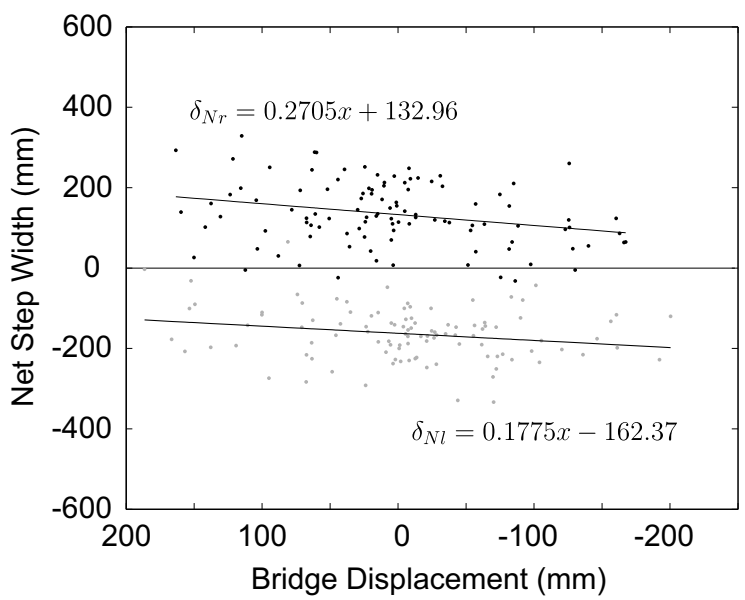

(a)

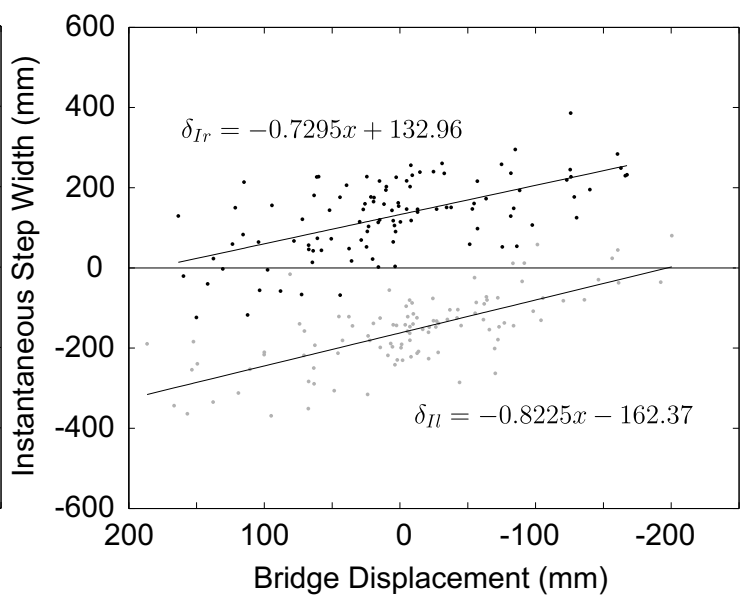

(b)

Figure 9: The (a) net and (b) instantaneous step width at heel-strike versus bridge displacement over the previous stance phase. Right-to-left steps (black) and left-to-right steps (gray). Positive width and displacement correspond to leftward motion; negative to rightward.

Note here that the subject did not appear to actively cross HS2 over TO1. Instead, the motion of the bridge caused the left foot to move under the swing foot. This implies a passive control of foot placement.

In Figure 8(e), a solid line parallel to the bridge nominally bisects the left and right heel-strikes into two parallel tracks. The left foot heel-strikes are all on the left side of the line, approximately equidistant from it, and the right foot heel-strikes are all on the right side of the line, approximately equidistant from it. The presence of these two distinct tracks is as if the subject were walking on a stationary surface because the heel-strike positions vary minimally from step to step. The NSW, measured as the lateral separation of consecutive heel-strike locations, is thus minimally affected by bridge motion.

In the same figure, the toe-offs are not consistently distributed about the line of walking. The dotted lines connect corresponding toe-off-heel-strike pairs, the lateral component of each representing ISW. These vary significantly along the sequence of steps, showing that instantaneous step width is heavily dependent on bridge motion. Thus, over the sequence of footsteps, the signs of the ISW starting with TO0 to HS1 are positive, zero (or slightly positive), positive, negative, and positive. In comparison, the four NSW signs starting with HS1 to HS2 are negative, positive, negative, positive. 


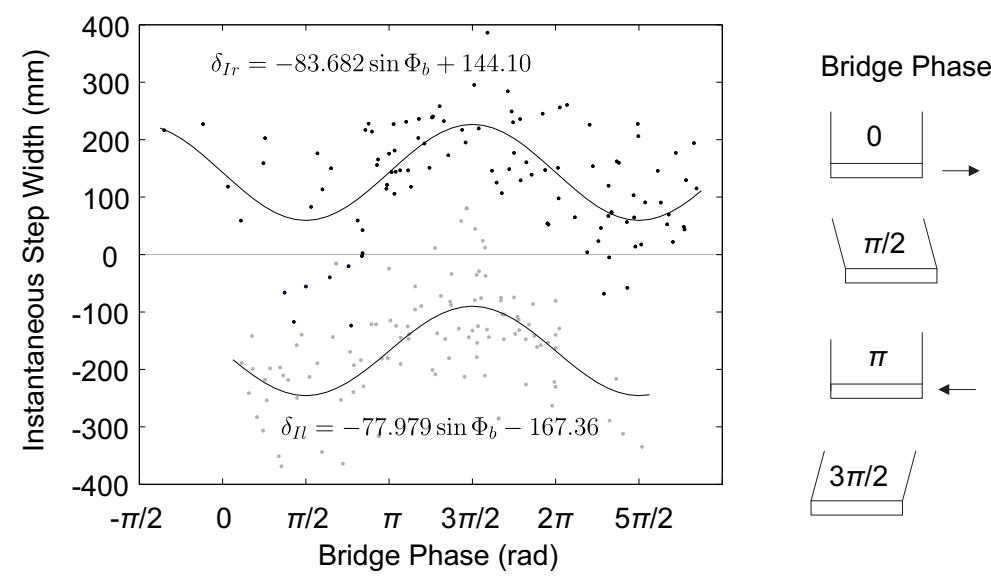

Figure 10: Instantaneous step width versus bridge phase. Right-to-left steps (black) and left-to-right steps (gray). Positive step width constitutes a leftward step; negative corresponds to rightward steps. Schematic shows frontal plane position (as seen from behind the pedestrian) and motion of bridge throughout one cycle.

Figure 9(a) depicts the NSW for 105 left foot-to-right foot and 105 right foot-to-left foot steps (henceforth 'left-to-right' or 'left-first/right-second' and vice versa) as functions of bridge displacement during stance. The linear regressions of the NSW are both less than 0.3, suggesting that the NSW was only somewhat dependent on bridge displacement. The $y$-intercepts were larger than the $91 \mathrm{~mm}$ stable ground step width identified by Macdonald [19], suggesting that walkers on a swaying bridge expand their stance width 40-70 $\mathrm{mm}$ on average compared to normal stable ground walking. Still, this modest step width expansion still constitutes landing the swing foot in a 'normal' position relative to the previous heel-strike step after step.

The ISW in Figure 9(b), however, exhibits a pronounced linear correlation with the structural displacement. This is to be expected with the inclusion of bridge motion in Equation 4. That said, the absolute value of the slope is less than one, indicating that some of the structural displacement is systematically mitigated by the pedestrian.

Comparing ISW to bridge displacement does not provide a wholly satisfactory result, however. The ISW itself is a snapshot of foot position, whereas bridge displacement describes a transition over time that considering an oscillating bridge - may be somewhat misleading. More fitting is a comparison of ISW with a snapshot of the state of the bridge. Hence, assuming simple harmonic motion, the phase of the bridge can be defined as its state (i.e. position and velocity) at heel-strike. The phases are defined such that phase $\pi / 2$ is the bridge's rightmost extent and $3 \pi / 2$ is the leftmost extent, both with velocity of zero. Comparisons can now be made between the phase of the bridge at a heel-strike (e.g. at $t_{1}$ ) and the subsequent ISW (at $t_{2}$ ) to investigate the implications of $\Delta_{x}$ on step width in Equation 4. Because of this aspect, each data point in the subsequent figures represents two states of the system: the phase of the bridge ( $x$-axis) at the first heel-strike, and the ISW ( $y$-axis) at the second heel-strike. This is the basis for the 'left-to-right' naming convention.

ISW turns out to be a sinusoidal function of bridge phase, as shown in Figure 10. The left-to-right and right-to-left foot offsets (-167.4 and $144.1 \mathrm{~mm}$ respectively) are similar to but higher than the stable ground step width proposed by Macdonald [19], again suggesting a slight widening of the stance. The ISW amplitudes are -78.0 and $-83.7 \mathrm{~mm}$ respectively.

Figure 11 shows the overall mean sinusoid with all the data. The sinusoid has been divided into quadrants corresponding to the bridge phase. Quadrant I depicts left-to-right ISWs for which the preceding left 


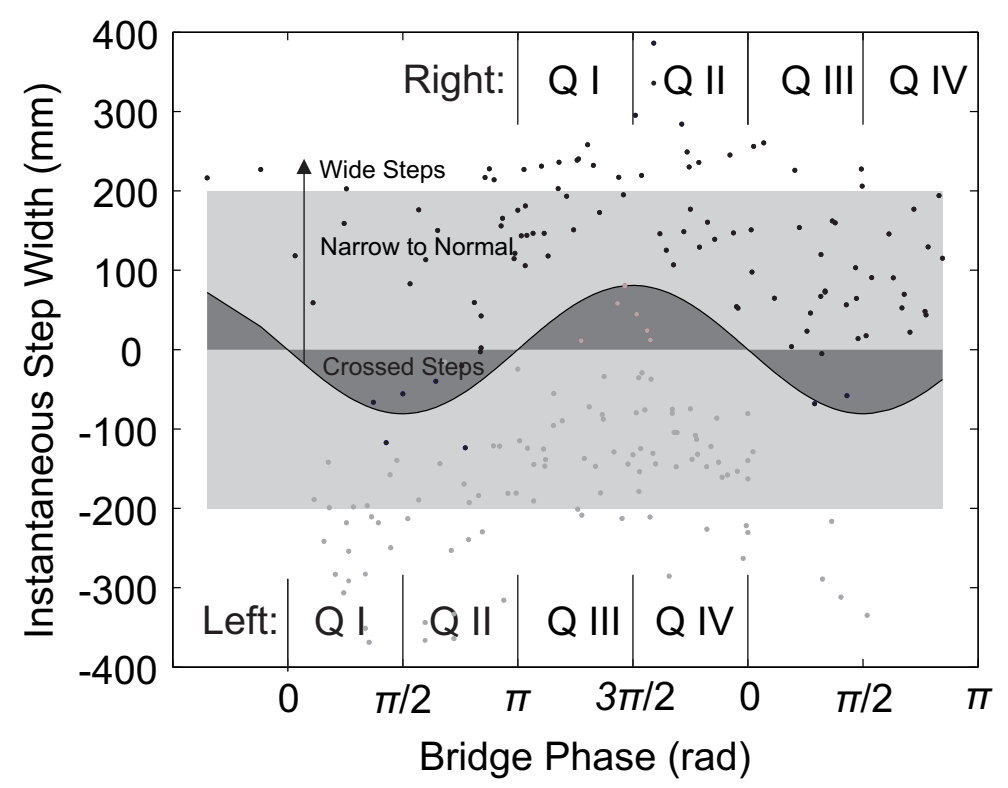

Figure 11: Step widths based on bridge phase at previous heel-strike. The mean of the two sinusoids in Figure 10 divides the left-to-right (gray) and right-to-left (black) footsteps. The sinusoid is divided into four quadrants, which are labelled according to the stance foot.

Table 2: Quadrants of HSI Based on Structural Characteristics and Heel-Strike Side

\begin{tabular}{lccccc}
\hline \multirow{2}{*}{ Quadrant } & Bridge Motion & \multicolumn{2}{c}{ Left-First Heel-Strike } & \multicolumn{2}{c}{ Right-First Heel-Strike } \\
& (Anatomically) & Bridge Position & Bridge Velocity & Bridge Position & Bridge Velocity \\
\hline I & Medially, Decelerating & Right & Right & Left & Left \\
II & Laterally, Accelerating & Right & Left & Left & Right \\
III & Laterally, Decelerating & Left & Left & Right & Right \\
IV & Medially, Accelerating & Left & Right & Right & Left \\
\hline
\end{tabular}

heel-strike occurs between bridge phases 0 and $\pi / 2$. Ergo the bridge is right of centre with rightward, decreasing velocity. Quadrant II is left-to-right ISWs for which the left heel-strike occurs between phase $\pi / 2$ and $\pi$ (bridge right with leftward, increasing velocity), and so on.

In contrast to left-first heel-strikes, Quadrant I for a right-first heel-strike starts at bridge phase $\pi$ instead of 0; the other quadrants are likewise shifted (Table 2). Thus, Quadrant I for left-to-right steps corresponds to Quadrant III for right-to-left steps and vice versa. While potentially confusing at first, this convention ultimately allows for easier anatomic reference. Quadrant I always describes the deck moving medially (i.e. towards the swing leg side) and slowing, whereas Quadrant III always describes the deck moving laterally (i.e. towards the standing leg side) and slowing. To aid in clarifying the use of quadrants, the immediate discussion will focus on left-to-right footsteps, but the behaviour is simply mirrored for right steps.

Quadrants I and II depict wide steps. The bridge is on the right side of centre, so a left-first heel-strike in this region is likely to be followed by a wide right step as the bridge swings back to the left before the second heel-strike. See the left-to-right step TO3 to HS4 in Figure 8 as an example. At HS3 the bridge was in QII, so therefore the ISW depicted in Figure 8(d) is a wide right step. The ISW model from Figure 10 peaks at approximately $\pm 250 \mathrm{~mm}$, but some data far exceed that width. The widest steps occur in QII, where the bridge and stance foot move laterally, away from the landing swing leg. Some step widths at the beginning of QI and the end of QII are narrow but they are never crossed. 
In Quadrants III and IV participants take the narrowest steps. The bridge is left of centre, so a left-first heel-strike is most likely to be followed by a narrow or crossed right step. This occurs because the bridge ultimately moves to the right, carrying the standing leg medially. Here the ISW model reaches its minimum in QIII and QIV at around $\pm 50 \mathrm{~mm}$, but the variation in step widths shows that some data take the opposite sign. Any points in Figure 11 that lie in the dark shaded regions show a heel-strike for which the next step crossed over. This has already been shown in the context of Figure 8, step TO1 to HS2; HS1 occurred at the start of QIV, so the resulting ISW was slightly crossed. This phenomenon exclusively occurs in QIII and QIV. Notice, however, that it does not always occur; most of the steps in this period are narrow or normal; not crossed over.

In summary, it has been shown that when walking on an oscillating surface, a subject's net step width is similar to his or her stable ground step width, regardless of the amount of base motion. This describes the overall strategy employed by the subject - walk in a straight line towards a destination - even when lateral oscillations are present and their feet move beneath them. It has also been shown that the instantaneous step width is a function of the bridge phase at the previous heel-strike. The quadrants of HSI were introduced to aid in understanding structure-stance relationship. This will be shown to correlate to the kinematics of gait and ultimately ground forces following an investigation of $\mathrm{CoP}$ and $\mathrm{CoM}$ trajectories.

Before continuing, it is worth mentioning a pair of promising recent investigations of step width by Carroll et al. [15] and Bocian et al. [16]. The works show strong evidence that treadmill walkers modulate their step widths by the so-called self-excited forcing frequency, $f_{\text {base }}-f_{\text {subject }}$, thus claiming to experimentally prove the accuracy of the IPM. While the trials in this paper are too short in duration to examine the self-excited forcing frequency, there is an unmistakable relationship between step width, bridge motion, and gait stability: the next apparent advancement will be to connect NSW (the pedestrian's overall 'strategy') to the self-excited forcing frequency via ISW, which incorporates structural and pedestrian motion.

It is noted, however, that Carroll et al. [15] propose an active step width strategy for foot placement. The Oxford data show, however, that mitigating the motion of a moving base is predominantly passive rather than active; ISW is mainly a passive by-product of the structural motion altering NSW. That said, the slight expansion of step width versus stable walking deserves attention, as does the stepwise variation of stride duration (which could dramatically alter ISW). These aspects of fine tuning may reflect the limited amount of active foot placement achievable by the subjects.

\subsection{CoP Displacement}

This section investigates the sum of the parameters $\Delta_{\mathrm{CoP}}+\Delta_{x}$ from Equation 4 . The goal here is to show how the CoP is affected by bridge motion between consecutive heel-strikes. Figure 12 shows CoP behaviour as a function of bridge phase, separated into bins of increasing structural amplitude. Subplots (a) and (b) show that when the bridge amplitude was low, Centre of Pressure displacements were less than $50 \mathrm{~mm}$ and the trajectories often followed the question mark ('?') shape typical of stable-ground walking [20]. In the first plot the samples were randomly distributed throughout the bridge cycle, but in the second plot, some of the right and left footsteps were clustered. Plots (c) and (d) - depicting bridge amplitudes of $25-50 \mathrm{~mm}$ and $50-75 \mathrm{~mm}$ - show that the CoP tracks began to align with the sinusoidal motion of the bridge. In the latter case, $\mathrm{CoP}$ displacements exceeded $100 \mathrm{~mm}$ in extreme cases. Finally plots (e) and (f) show CoP displacement for the greatest structural amplitudes.

In the last two plots the CoP displacement was generally dominated by the motion of the bridge. When the bridge was at its maximum extents at phases $\pi / 2$ and $3 \pi / 2$, the right or left (respectively) CoPs reflected the structure's sinusoidal motion. As the structure moved back towards centre, the standing foot did so as well. This 'convergent' behaviour compressed the separation between CoM and CoP, and could have been a source of positive damping or negative work on the bridge. Around the inflection points $\pi$ and $2 \pi$, however, 


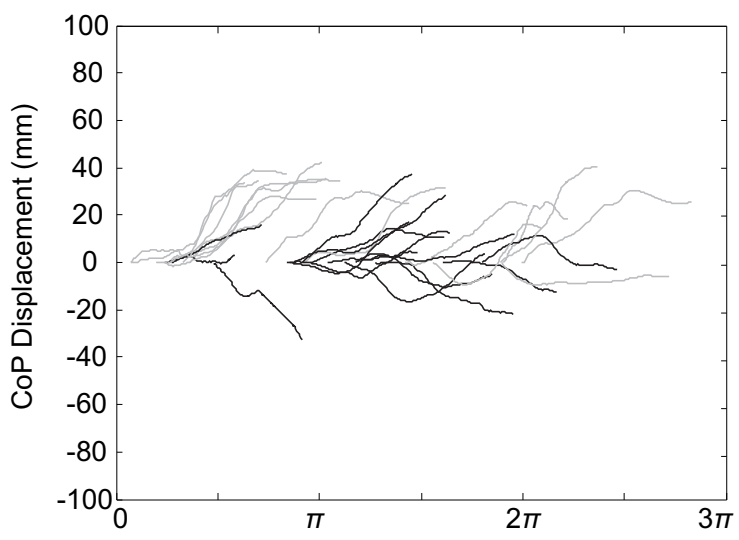

(a)

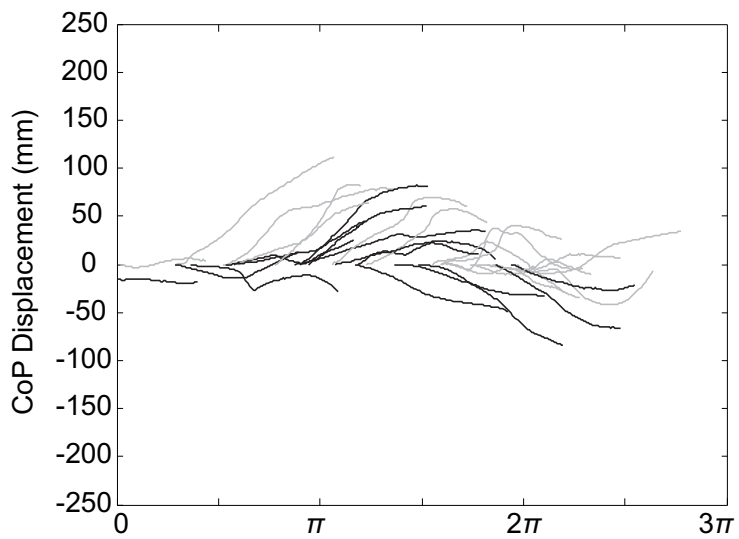

(c)

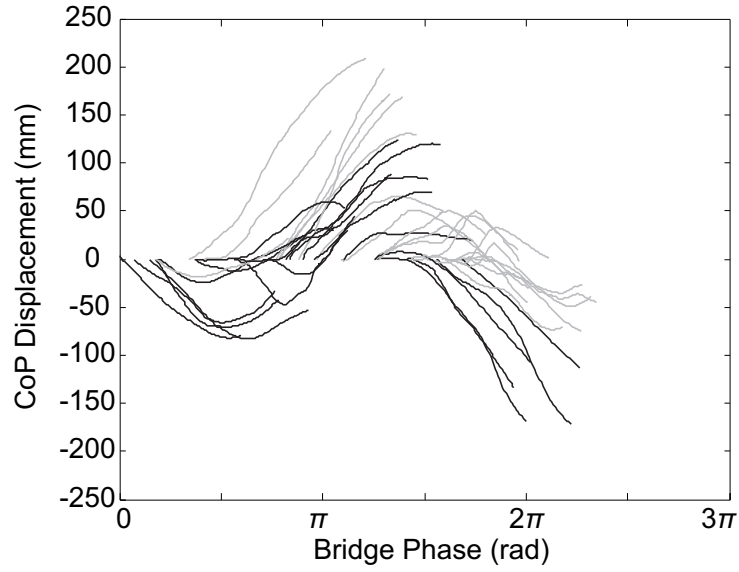

(e)

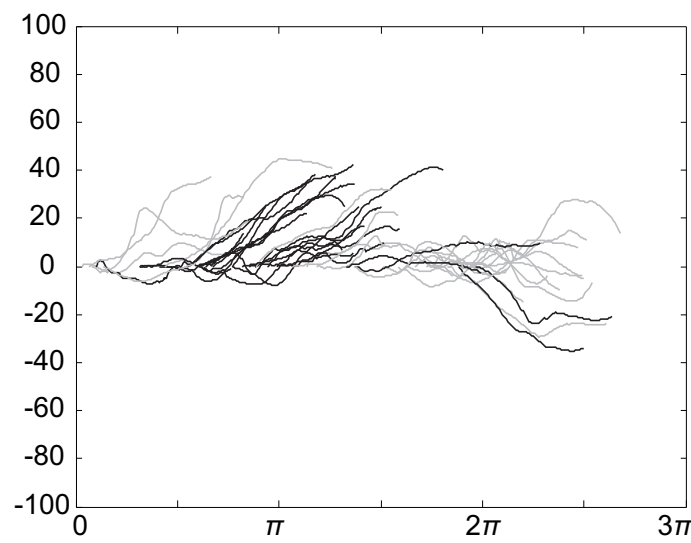

(b)

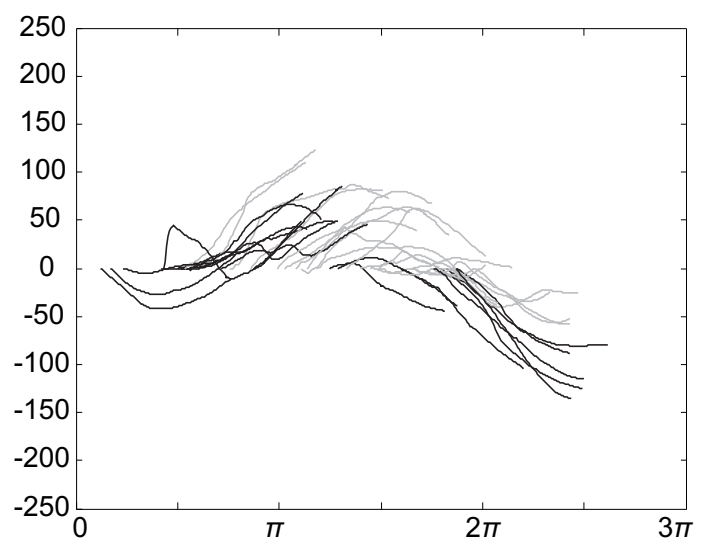

(d)

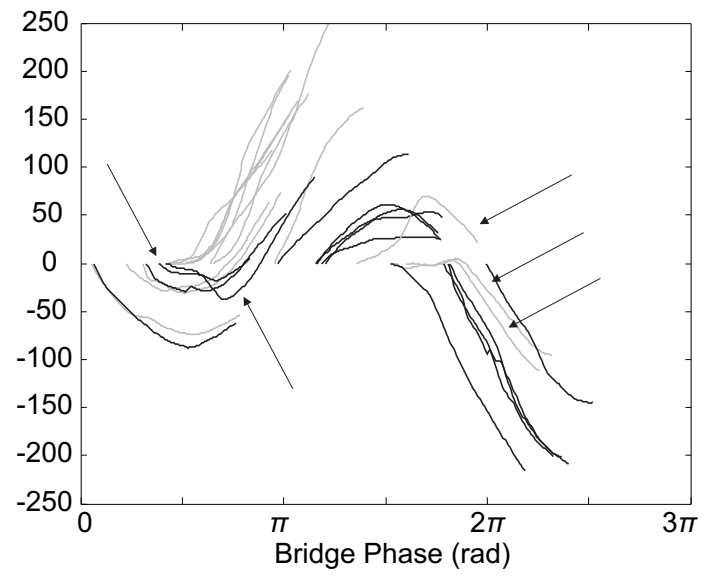

(f)

Figure 12: Centre of Pressure displacement during single stance versus bridge phase for different ranges of bridge amplitude. (a) Amplitude < $12.5 \mathrm{~mm}$, (b) $12.5-25 \mathrm{~mm}$, (c) $25-50 \mathrm{~mm}$, (d) $50-75 \mathrm{~mm}$, (e) $75-100 \mathrm{~mm}$, and (f) 100-125 mm. Left (gray) and right (black). 


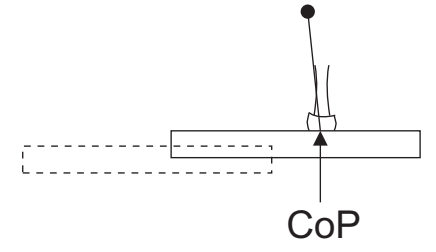

(A)

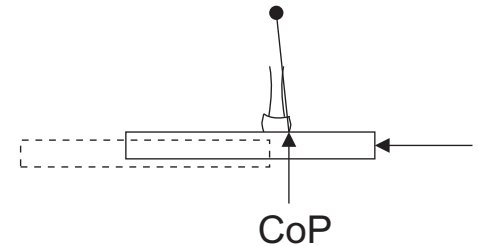

(B)

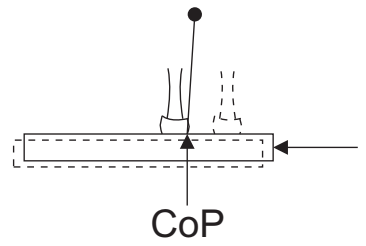

(C)

Figure 13: A right foot stance phase (frontal view from behind) with bridge at ipsilateral extent. As (A) stance phase begins, bridge velocity zero; CoP normal. With (B) bridge acceleration towards centre, CoP moves to right extreme, where it becomes bridge-controlled. If (C) the CoP displacement cannot compensate for CoM motion, the subsequent left step (dashed) is crossed.

the left and right CoPs (respectively) appear to be somewhat tangential, projecting away from the sinusoidal structural motion. This 'divergence' took the stance foot laterally, away from the Centre of Mass, and could have resulted in a pedestrian providing negative damping or positive work to the structure, accompanied by a lateral push-off.

Some samples, such as the curves indicated with arrows in Figure 12(f) are not fully concentric with the structure's sinusoidal motion. The indicated two right CoPs and three left CoPs (all starting late in QIII) begin with a period of zero CoP displacement. They then moved laterally to a maximum before reversing medially and aligning with the concentric curves. In these situations, single stance started when the bridge velocity was almost zero. Thus, the zero CoP displacement suggests that the subject's CoP briefly acted in a stable base manner. As the bridge started moving towards the centre, the CoP swung laterally in an attempt to compensate for the motion of the structure. At the maximum $\mathrm{CoP}$ displacement, the $\mathrm{CoP}$ reached the outside edge of the foot, the limit of the Base of Support. At this point it became controlled by the motion of the bridge and swung medially, conforming to the bridge's sinusoidal motion. This condition also implies that in order to maintain gait stability, the subsequent foot crossed over to ensure that the CoM remained within the Base of Support (Figure 13).

In summary, four Centre of Pressure behaviours were present when subjects crossed the moving base. First, when base motion was less than $25 \mathrm{~mm}$ amplitude, CoP trajectories were largely unaffected by base motion. Second, CoP motion for amplitudes over $25 \mathrm{~mm}$ generally reflected the sinusoidal oscillations of the bridge. Third, for steps taken during Quadrant II at high bridge amplitudes, the CoP displacement diverged from sinusoidal, resulting in a high instantaneous step width. Fourth, steps taken late in Quadrant III exhibited a moment of normal stable ground gait before contralateral bridge motion controlled the CoP, causing a narrow or crossover step.

\subsection{CoM Displacement}

Unlike the ISW and CoP, the Centre of Mass motion exhibited by the subjects did not readily correspond to base motion. Nonetheless, the subjects did overwhelmingly appear to mitigate the motion of the base and keep their CoM stable.

Brady et al. [25] described how subjects on a laterally oscillating treadmill adopted one of two strategies for maintaining stability. Fixed-in-space (FIS) walking is achieved by relaxing the body, allowing the feet to sway beneath a stationary CoM, which acts as a pivot. In contrast, in fixed-to-base (FTB) walking, the subject stiffens his or her muscles, causing the body (ergo $\mathrm{CoM}$ ) to move monolithically with the bridge. Subjects can switch between strategies, although doing so may cause the subject to lose their balance.

In the Oxford tests, it was difficult to assess whether subjects adopted a FIS or FTB strategy for bridge ranges under $50 \mathrm{~mm}$ because normal CoM ranges are of the same magnitude. Even at larger amplitudes, 
Table 3: Mean and SD CoM Ranges for Different Bridge Ranges.

\begin{tabular}{lccc}
\hline Bridge Range $(\mathrm{mm})$ & $n$ & Mean CoM range $(\mathrm{mm})$ & SD CoM range $(\mathrm{mm})$ \\
\hline $0-50$ & 37 & 61.3 & 21.5 \\
$50-100$ & 15 & 68.9 & 37.4 \\
$100-150$ & 19 & 79.8 & 24.3 \\
$150-200$ & 20 & 80.7 & 32.4 \\
$200-250$ & 14 & 85.4 & 33.8 \\
All Samples & 105 & 72.6 & 29.6 \\
\hline
\end{tabular}

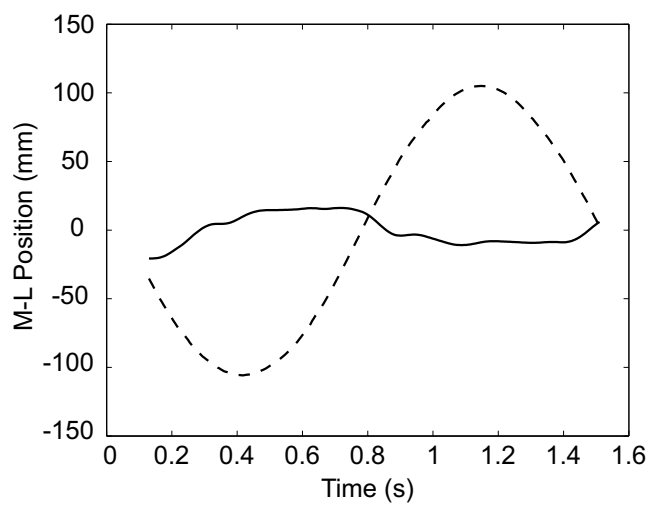

(a)

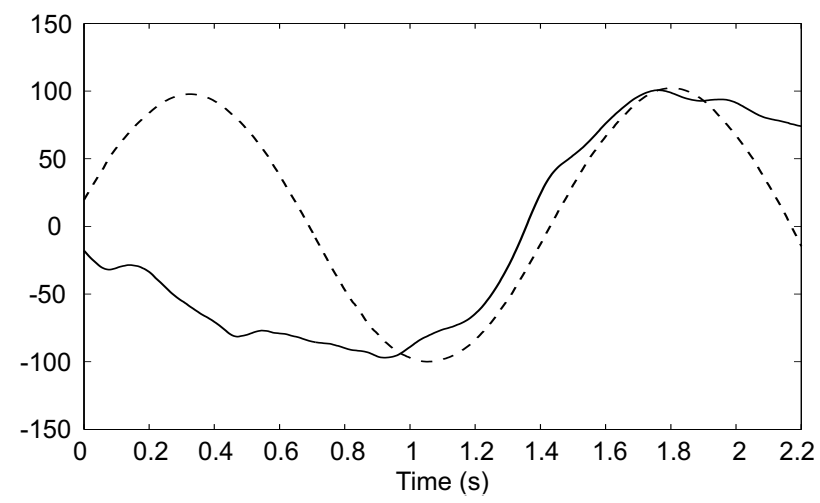

(b)

Figure 14: Examples of (a) fixed-in-space CoM strategy versus (b) transient fixed-to-base strategy. CoM (solid) and bridge position (dashed) versus time.

there was only a weak relationship between bridge amplitude and CoM range (see the high standard deviations in Table 3), but visual inspection of individual trials allows for the categorisation of different CoM strategies. Omitting the low-bridge amplitude trials, 65 of the remaining 68 trials exhibited a FIS strategy. In these, the CoM trajectory neither matched the amplitude nor the sinusoidal shape of the bridge motion throughout the three-step trial. The remaining three trials appeared to be purely FTB.

Curiously the study by Brady et al. [25] produced opposite results to the Oxford results, finding that their participants were largely FTB. Their laterally oscillating treadmill had a peak-to-peak displacement of $254 \mathrm{~mm}$, comparable to the maximum bridge displacement in this study, but they tested two oscillation frequencies, 0.2 and $0.3 \mathrm{~Hz}$, which were less than half of Oxford's. For the same amplitude, the lower frequency imparts a lower lateral acceleration on the pedestrian, which would be easier to accommodate with a fixed-to-base strategy.

One behaviour that was observed in the Oxford tests was transient FTB strategy. In 20 of the 65 FIS trials, the subject appeared to momentarily fix his or her CoM motion to that of the bridge. An example of this may be seen in Figure 14, where one plot shows a FIS trajectory by base motion while the second shows the CoM temporarily locking-in with the bridge motion. No Oxford subject maintained a transient FTB strategy for longer than half of one bridge cycle, suggesting that the lateral acceleration was too great for the subjects to remain fixed to.

Nonetheless, where some Oxford subjects adopted a transient FTB strategy by locking-in with the structural motion, this phenomenon should be investigated further. Most early theories regarding crowdinduced bridge excitation suggested human-structure footstep lock-in as a primary cause $[2,9,12,26]$. While this assertion is now more contentious, it remains a possibility that CoM lock-in (i.e. FTB strategy) could have an effect (or at least have played into the development of the theories). 


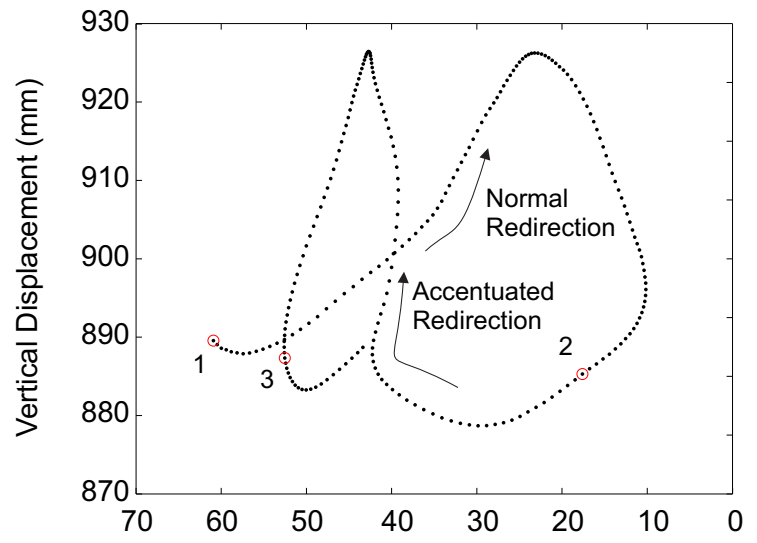

(a)

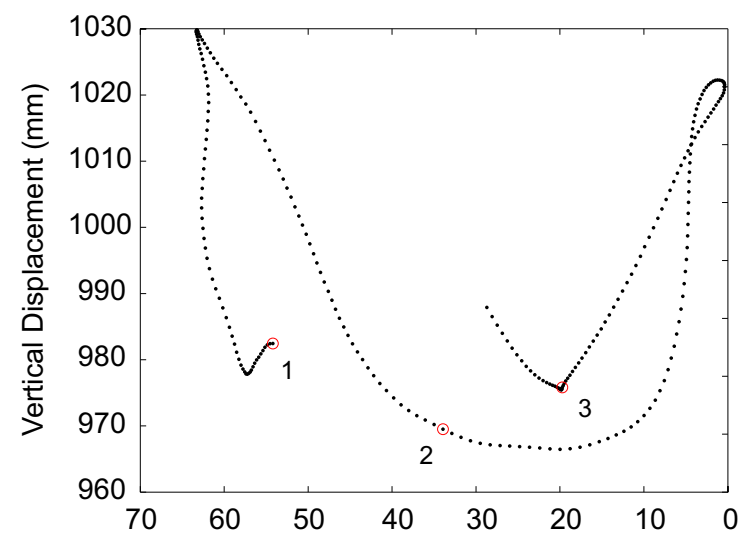

(c)

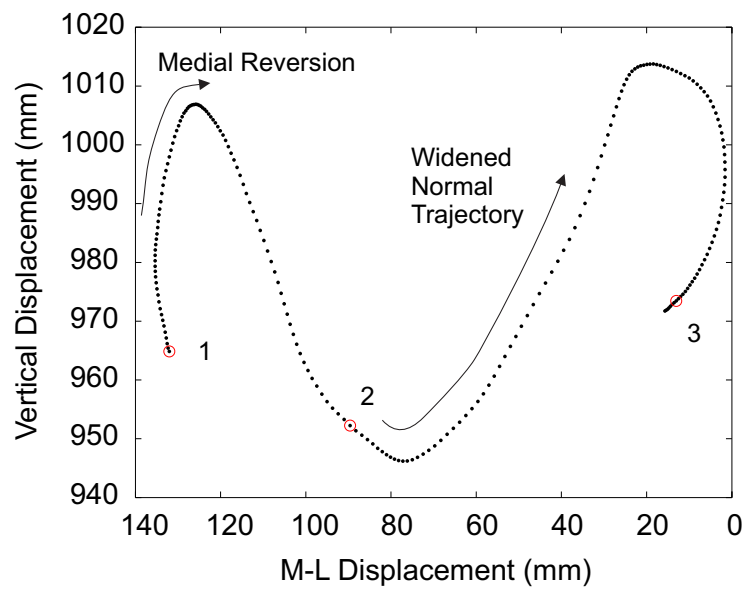

(e)

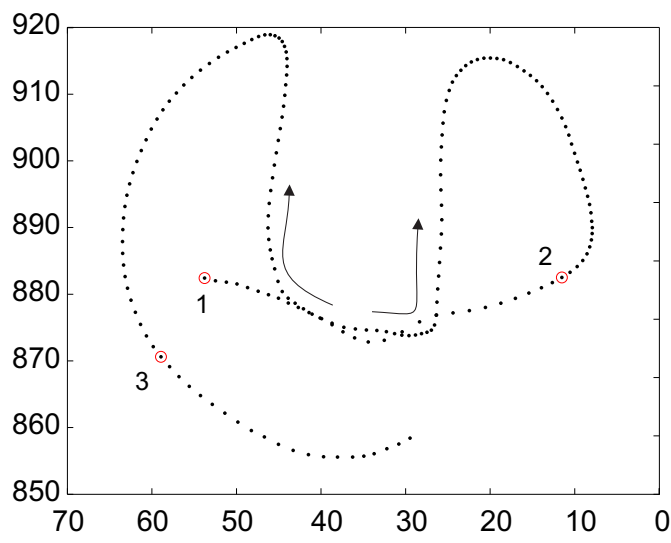

(b)

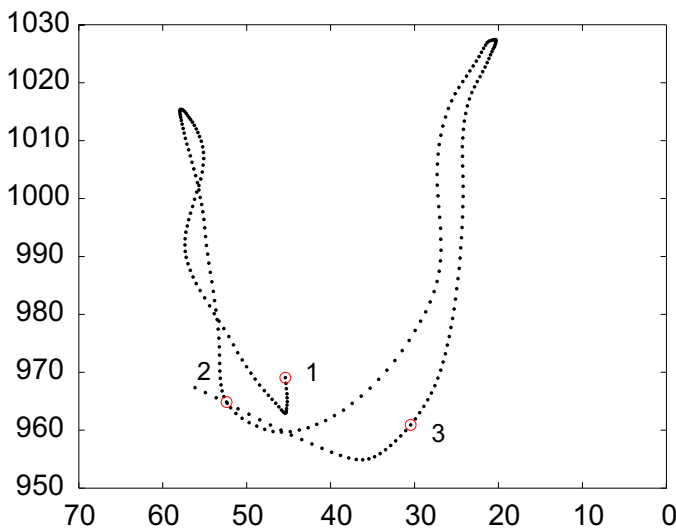

(d)

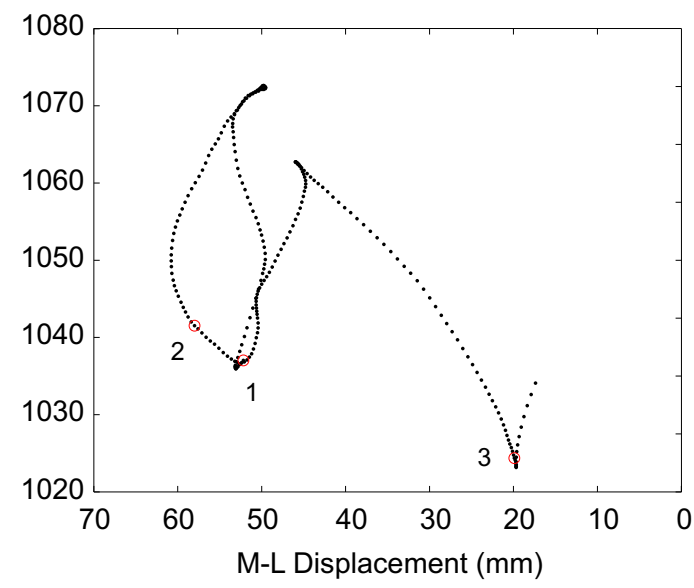

(f)

Figure 15: Two-step CoM trajectories for coping with structural motion. Frontal plane projections from behind with heel-strike points circled. (a) Near normal trajectory; (b) accentuated vertical redirections; (c) \& (d) U-shaped trajectory; (e) wide trajectory; and (f) eccentric trajectory. See text for discussion. 
But this discussion now turns to some of the more subtle strategies employed by test subjects to mitigate base motion, evidenced by the CoM frontal plane trajectory. Unlike the conscious selection of FTB or FIS strategy, these patterns appeared to be reactive, perhaps subconscious. Figure 15 shows a couple of the most commonly observed CoM patterns, which occurred for bridge amplitudes over $75 \mathrm{~mm}$.

Figure 15(a) shows a figure-eight trajectory similar to those typical of normal over-ground walking [27]. Even though the bridge amplitude is high, it shows that normal CoM trajectories are possible on a moving base. That said, it and Figure 15(b) exhibit sudden redirections of the CoM after heel-strike, indicated with arrows. During this behaviour the CoM changes from lateral to vertical motion at a near right angle. A mild alteration of CoM trajectory can occur in normal walking, but the behaviour was more pronounced and more common in moving base trials. The change in CoM direction does not correspond to toe-off timing, so it is possibly due to an acute, corrective rise through the knee and/or ankle.

In many cases, subjects had a U-shaped CoM trajectory during the moving base tests, as observed in Figure 15(c) and (d). The total lateral CoM range in these cases remained small compared to the bridge amplitude. Most of the lateral motion occurs immediately after heel-strike, while most of the vertical motion transpires during single stance. These patterns show that the CoM retraces its trajectory, rather than making a figure-eight. When M-L position is plotted against time, these U-shaped trajectories are manifested as flattened sinusoids, which will be shown later.

Though not the majority, there were cases of significant lateral displacement, such as during transient FTB motion. Figure 15(e) shows an example. In the first step vertical motion causes a medial reversion of the CoM path. The subsequent step is corrected, but the medially directed motion of the first step causes the trajectory of the second step to be slightly wider than normal.

Finally, 15(f) shows an example of an irregular CoM trajectory. These could have been caused by body rotation about the vertical axis, sudden applications of upper body angular momentum, or other stabilising motions.

Even though the aforementioned trajectories are subtle, abrupt changes to CoM trajectory develop high accelerations. Though in a physical sense these levels would still be small compared to what humans can cope with, ground force models such as the Inverted Pendulum Model [28] and Extrapolated Centre of Mass [29] depend on the accurate measurement or prediction of location, velocity, and acceleration parameters.

The variety of CoM trajectories presented here highlight the various ways individuals manage gait instability. Even though the lateral CoM motion is usually small for fixed-in-space walkers, patterns clearly vary depending on the individual. Given that the IPM relies on CoM-CoP lateral separation, the relationship of these two parameters will be investigated in the next section.

\subsection{CoM-CoP Separation}

Figure 16 shows how the CoM-CoP lateral separation is affected by bridge phase and amplitude. At low bridge amplitudes the CoM-CoP separation is not visibly affected by the phase of the bridge. The $\mathrm{CoM}$ is always within $150 \mathrm{~mm}$ of the CoP. The trajectories are all relatively flat with a few low-amplitude oscillations in the early to middle portion of the step.

For the higher bridge amplitudes, phase dependence is observed. The CoM-CoP separation in QI is sinusoidal with the least separation occurring when the bridge was at its contralateral extent $(\pi / 2$ for left feet, $3 \pi / 2$ for right). In some cases the CoM-CoP separation passed zero, suggesting that the the stance foot passed underneath the CoM. On a stationary base, this would cause lateral gait instability, but the acceleration of the stance foot quickly reverts the CoP to the 'normal' side of the CoM.

For stance phases beginning in Quadrant II, divergent CoM-CoP separation is observed, which corresponds to the CoP patterns discussed previously. The separation was maximised in this phase as the stance foot moved up to $250 \mathrm{~mm}$ away from the CoM. 

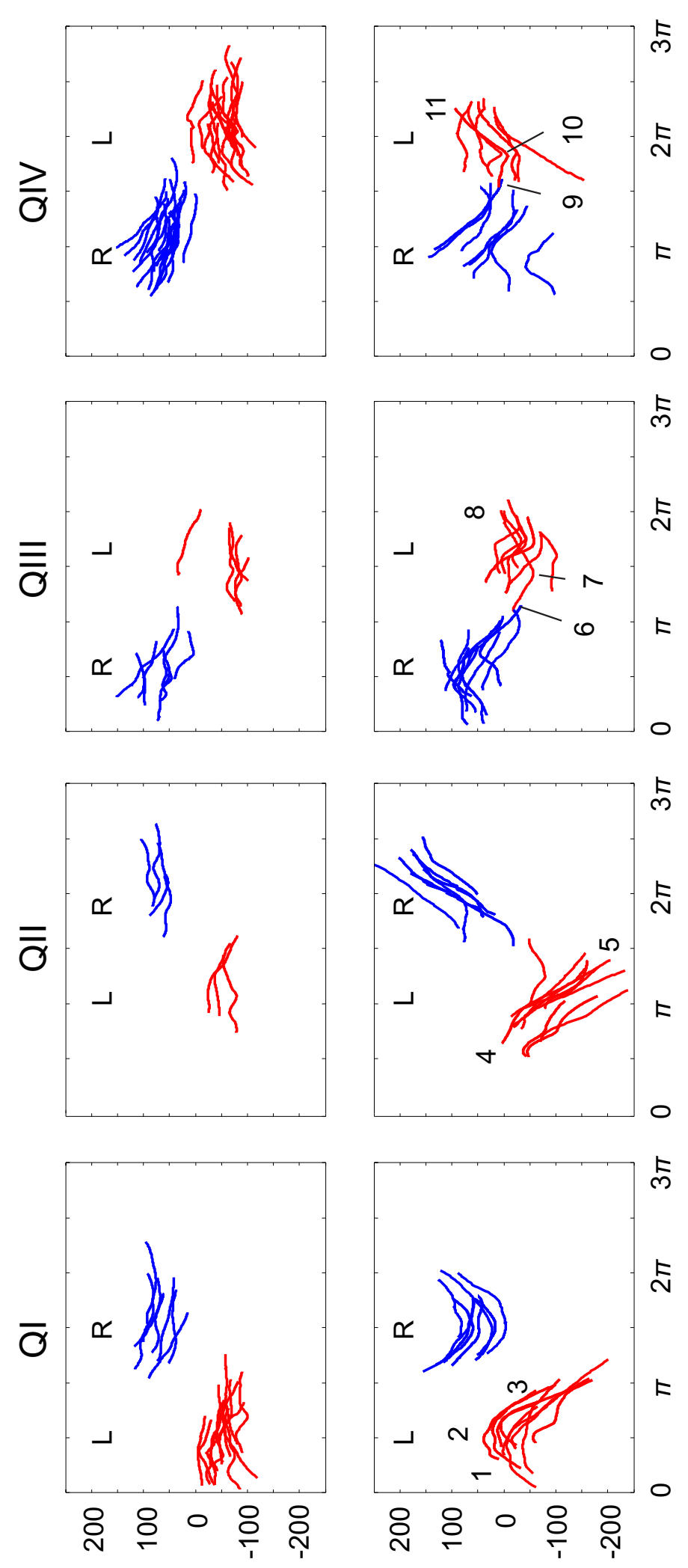

(mim) doว-Woว

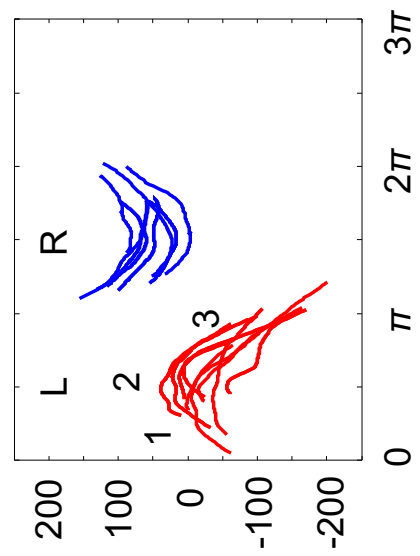

(mim) doว-พоว

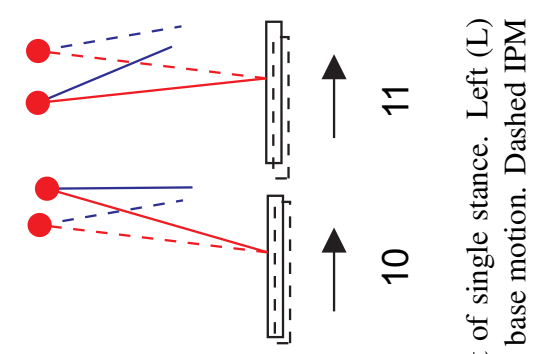

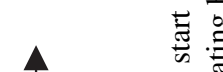

$\uparrow a \quad \frac{\pi}{0}$

$\frac{0}{2}$

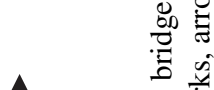

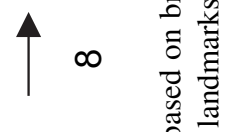

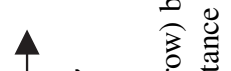

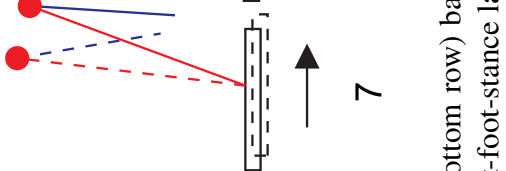

흥
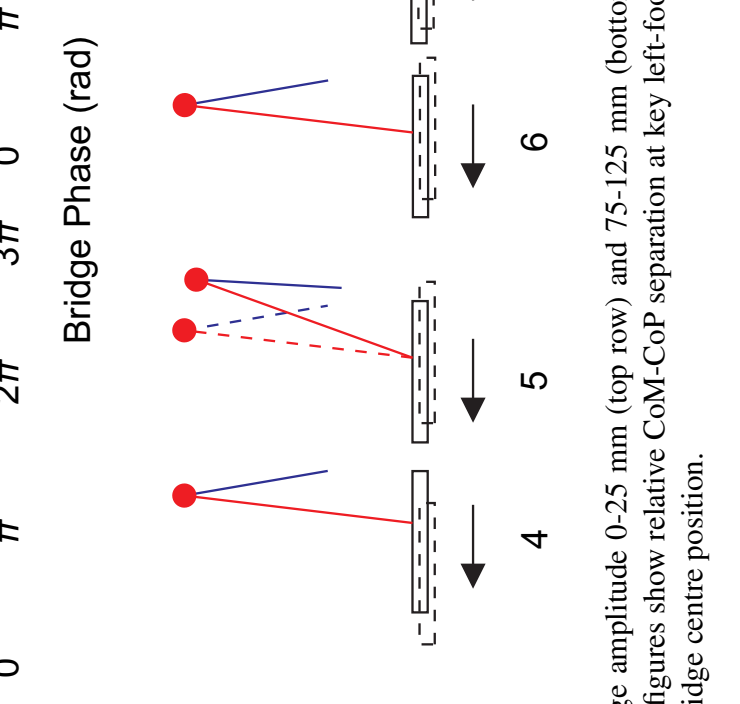

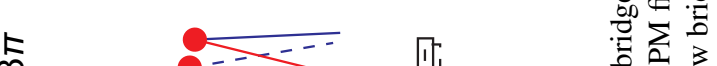
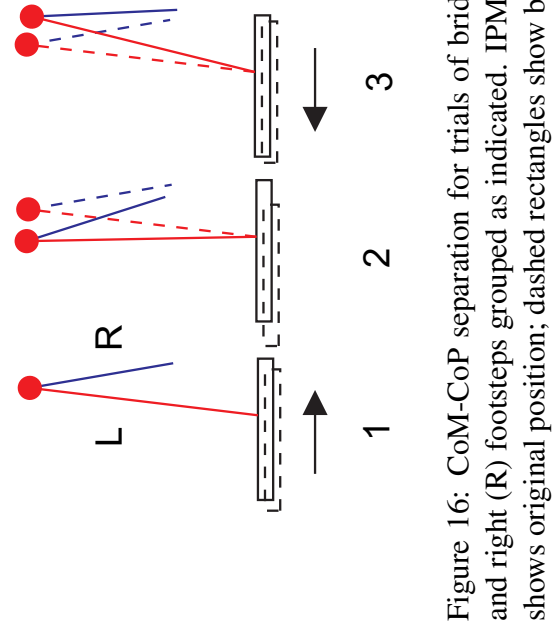


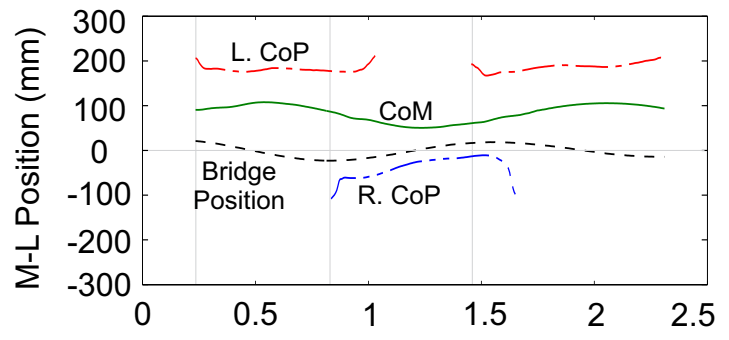

(a)

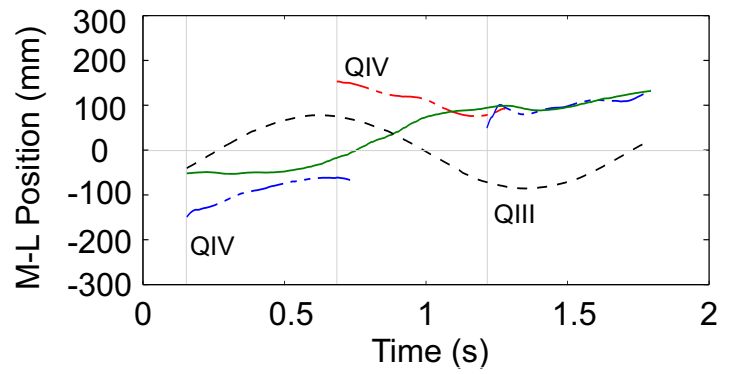

(c)

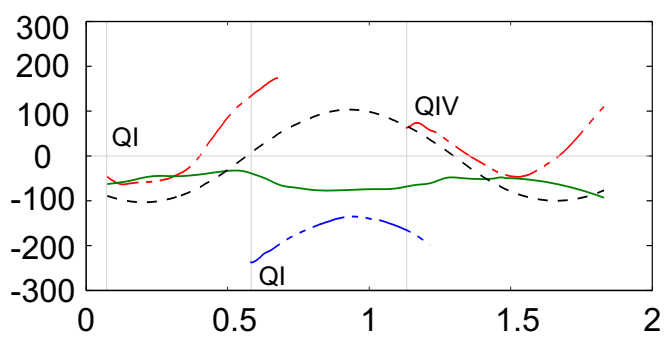

(b)

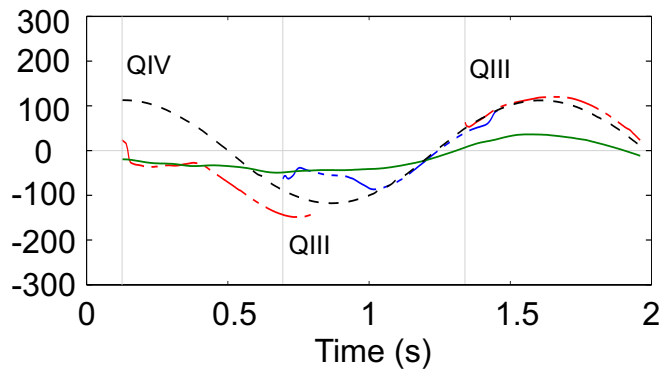

(d)

Figure 17: The relationship between CoM (solid), CoP (dash-dot (left) or dash-double dot (right)), and base motion (black dashed) for different phases of the structure. Grid indicates heel-strike times. See text for discussion.

Where the patterns in QI and QII were generally concentric, those of QIII and QIV are less so. Quadrant III reflects a similar behaviour to that mentioned for the CoP. In most cases the CoM-CoP separation increases slightly to a peak just after the bridge reaches its ipsilateral (e.g. leftward for left foot) extent. After the peak the CoM-CoP separation decreases, sometimes appearing to follow a sinusoidal trajectory.

Finally Quadrant IV reveals predominantly converging behaviour with the highest proportion of crossed footsteps. Most of the trajectories tend towards zero, indicating that the CoP is moving closer to the CoM. This is logical given that CoM and CoP are moving in opposite directions to each other at heel-strike ${ }^{2}$. Alternatively, some samples have a peak in early to mid-stance in the abnormal direction (indicators 9-11). This suggests that the CoM initially falls ahead of the CoP (expanding the CoM-CoP separation) before reverting and crossing laterally over the CoP.

Now it is beneficial to examine the CoM and CoP holistically over several steps. Figure 17 shows threestep CoP traces with the bridge position and CoM location as a function of time. In Figure 17(a), where the bridge motion is small, the CoP traces are shaped normally and spaced evenly. The CoM oscillates in a quasi-sinusoidal manner, out of phase with the bridge. Figure 17(b) shows a subject walking nearly in phase with the bridge. The first, left, step begins at the end of QI (indicated by the amplitude and slope of the dashed line), resulting in divergent behaviour and a wide second step. The right step also begins in QI and leads to a wide third step. The final step begins in QIV and initially tracks the motion of the bridge. The CoM has a flattened sinusoidal trajectory, clearly shifting from left to right and vice versa but remaining relatively linear during single stance. This is indicative of the U-shaped frontal plane pattern identified previously and is a FIS strategy. Figure 17(c) depicts a crossed step. With the first (right) single-stance

\footnotetext{
${ }^{2}$ Claff [23] confirmed experimentally that on stable ground the CoM moves laterally from departing foot towards the receiving foot during double stance. On a moving bridge in QIV, the receiving foot moves medially towards the CoM.
} 


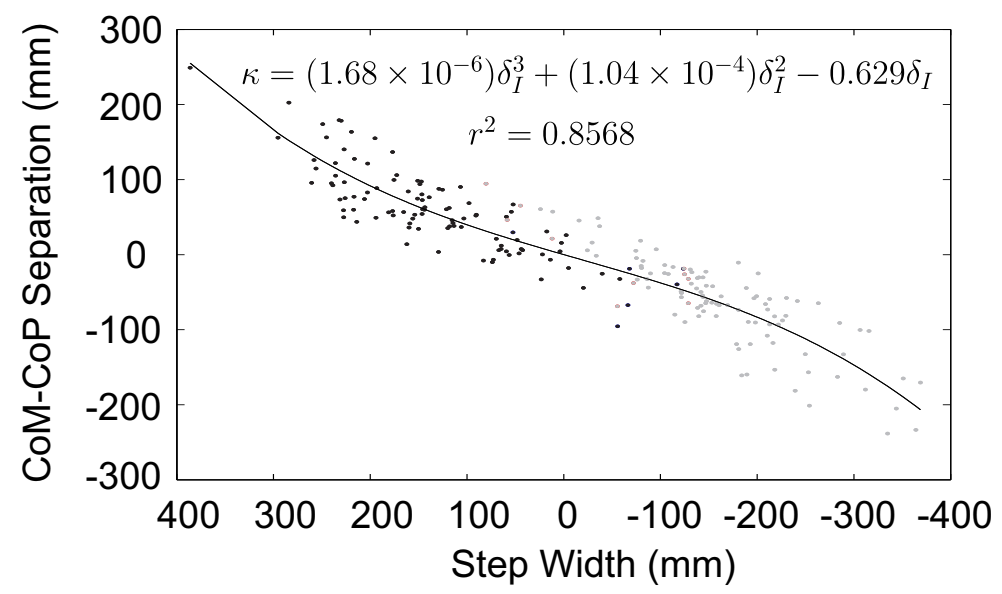

Figure 18: CoM-CoP separation as a cubic function of ISW. Left-to-right steps (gray) and right-to-left steps (black).

beginning at the start of QI (although the heel-strike occurs at the end of QIV), the second step is a wide left step. This left step begins in QIV, so the subsequent right crosses over the previous step. The CoM is normal for the first step, but crosses the CoP in the second step and follows the CoP in the third. The final frame, Figure 17(d), shows a left and a right step beginning in QIV and QIII respectively. They have flat regions before conforming to the sinusoidal motion of the bridge. The second step is crossed and the final step is in line with the second (i.e. ISW $=0$ ). The CoM is flat for the first two steps before adopting a slightly wider trajectory for the third step.

Studies such as that by Oddsson et al. [30] have suggested that M-L ground force is proportional to ISW. Thus, the step widths shown in Figure 17 become of importance to M-L ground force prediction. The CoM-CoP separation was plotted against ISW to see if a relationship existed between the variables at heelstrike. Figure 18 shows that the CoM-CoP separation for both left-to-right and right-to-left is fitted well by a single cubic function. The CoM-CoP separation of the receiving foot is best fitted by the equation

$$
\kappa=\left(1.68 \times 10^{-6}\right) \delta_{I}^{3}+\left(1.04 \times 10^{-4}\right) \delta_{I}^{2}-0.629 \delta_{I}
$$

where $\kappa$ is the CoM-CoP separation.

In summary, the CoM-CoP separation was found to be similar to the CoP trajectories because the fixedin-space CoM was small. Over consecutive steps, the CoM-CoP separation was rendered a cubic function of the ISW and therefore related to the bridge phase. As forces and modelling are investigated in the next section, it will be shown that CoM-CoP separation is the primary component of M-L ground force, therefore requiring proper measurement and prediction of $\mathrm{CoM}$ and $\mathrm{CoP}$ trajectories.

\subsection{Force Patterns $\mathcal{E}$ Modelling}

Thus far a number of patterns have been identified regarding the placement of the foot, the CoP trajectory, the CoM trajectory, and CoM-CoP separation. These kinematic data are plotted with the ground forces and the simulated Inverted Pendulum Model in Figure 19. The eight trials depicted represent the most relevant and common characteristics across the 105 trials and will be discussed in depth in the following sections.

The bottom graph of each set provides the recorded and calculated (IPM) M-L ground force. The IPM is a simple model relating M-L bridge and CoM accelerations to a pedestrian's CoM-CoP separation. It assumes that the $\mathrm{CoP}$ is fixed for each stance phase and that a pedestrian can be modelled as a rigid inverted 

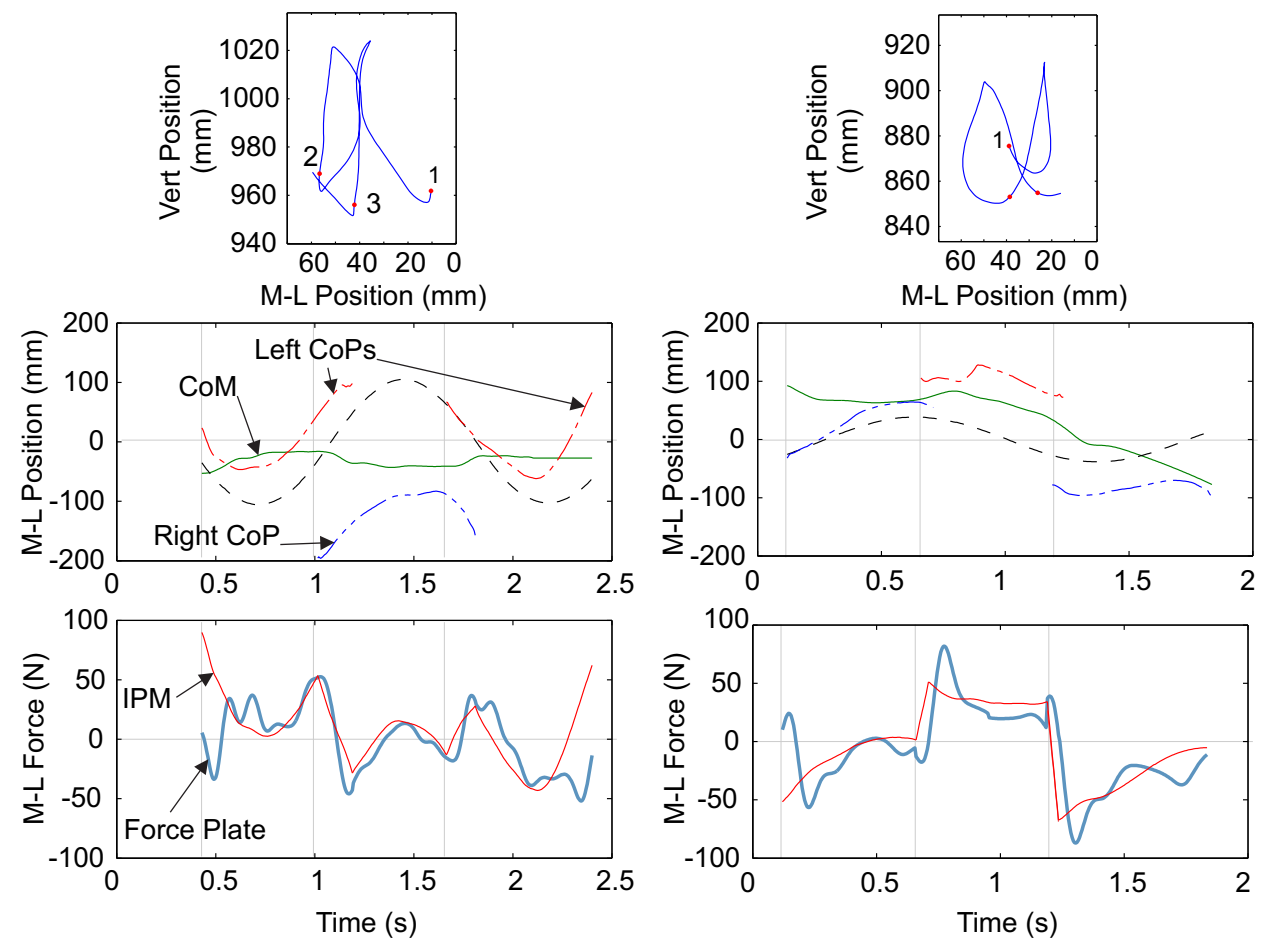

(a)

(b)
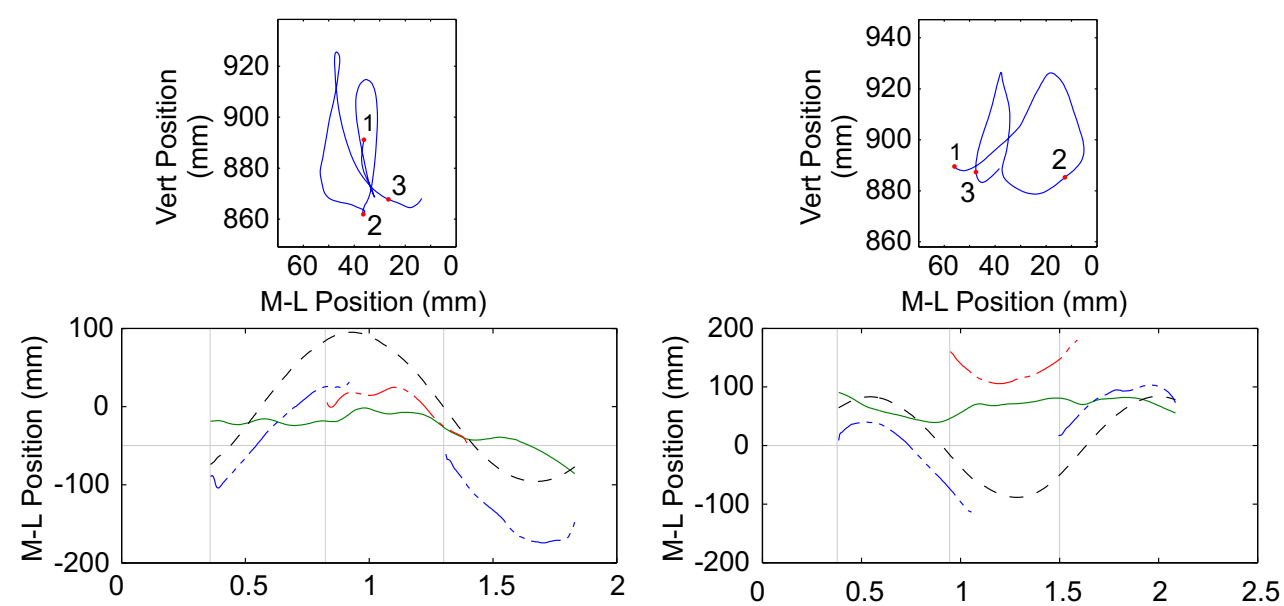

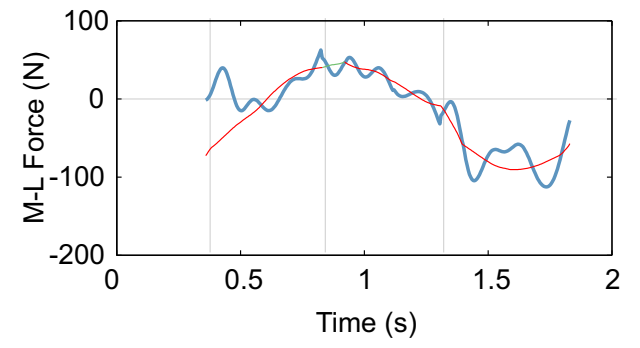

(c)

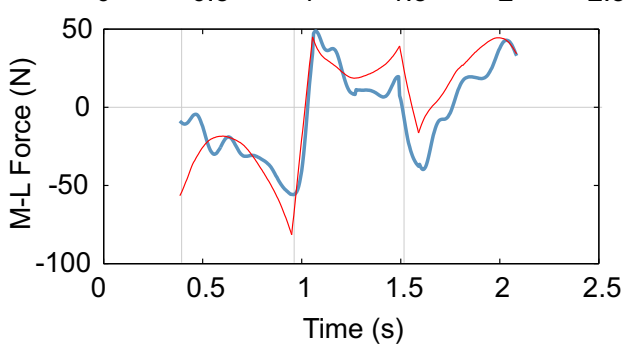

(d)

Figure 19: (Continued next page) 


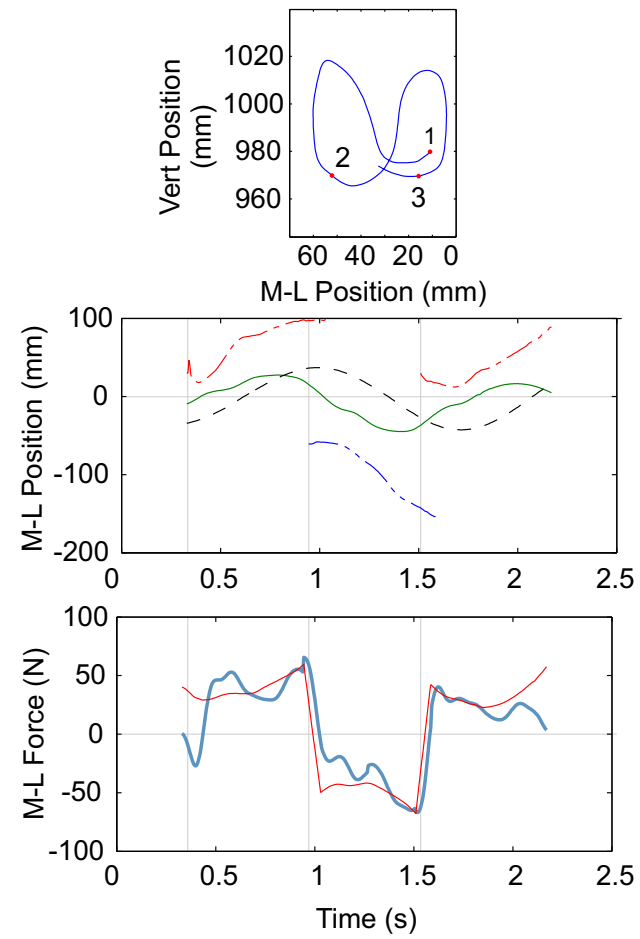

(e)
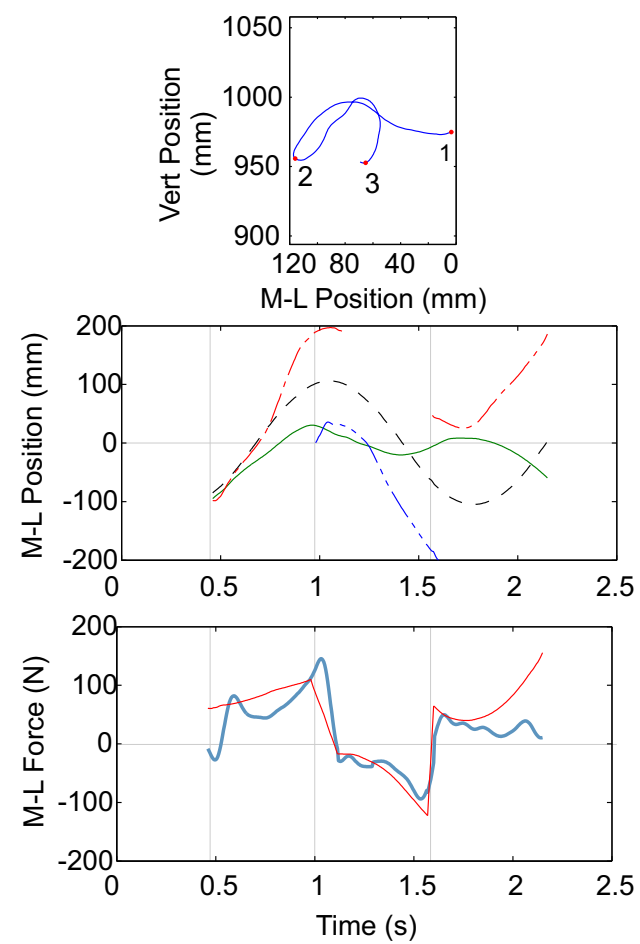

(g)
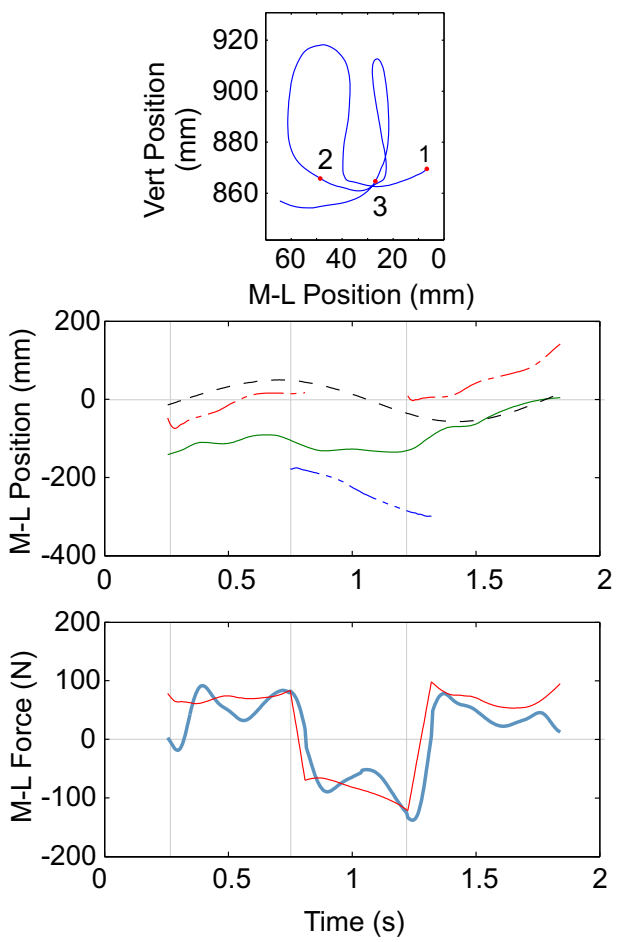

(f)

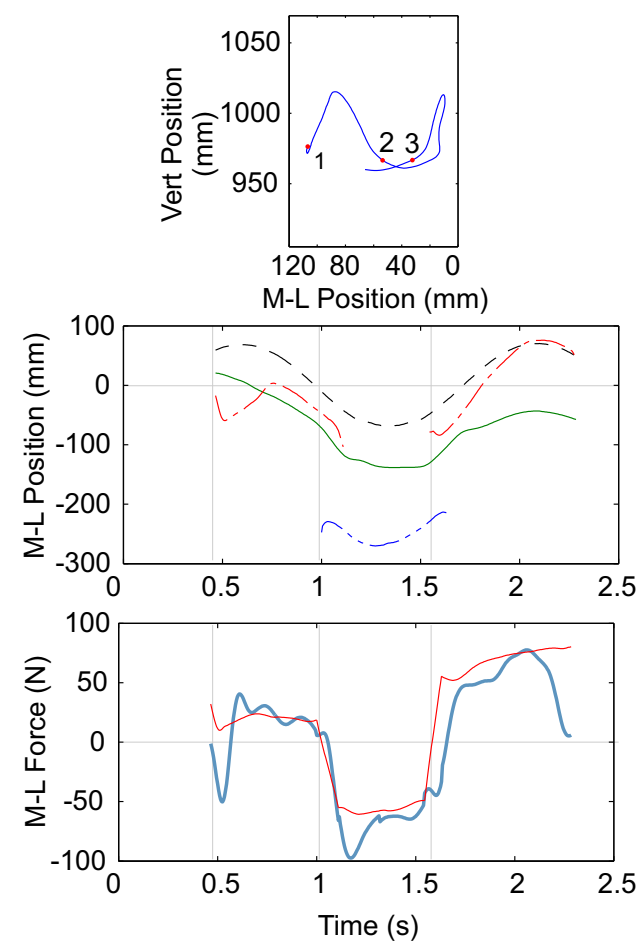

(h)

Figure 19: Top: Adjusted CoM trajectory (first two steps), heel-strikes indicated. Middle: Bridge position (black dash), CoPs (left dash-dot, right dash-double dot), CoM (solid). Bottom: Sum of force plates (bold), and IPM (solid). Grid lines mark heel-strike times. See text for discussion. 
pendulum. See Macdonald [19] for details. For the IPM calculations here, the pendulum length was selected as the distance from the $\mathrm{CoM}$ to the $\mathrm{CoP}$, though using a constant $1200 \mathrm{~mm}$ gave similar results. Also, the CoP-CoM separation $(u-y)$ was chosen to be the difference in the lateral positions between the toe marker and the CoM rather than between the true $\mathrm{CoP}$ and the $\mathrm{CoM}^{3}$. Finally, the ground force has been linearly interpolated from one foot to the next over the duration of double stance.

\subsubsection{Force Plate Results}

When examining the force plate records, the first aspect that stands out is that each record is unique. The eight records are not only produced by different individuals, but they occur at different bridge phases. Nonetheless, some general patterns still emerge.

The amplitude of the ground forces is generally correlated to the CoM-CoP separation. This trend is particularly evident in cases where the CoM-CoP separation is small. In Figure 19(a) steps one and three, the left CoPs touch the CoM trajectory, resulting in a lateral ground force close to zero. Similarly in Figure 19(b) the right CoPs approach the CoM, so M-L force decreases from (negative) $50 \mathrm{~N}$ to zero. In Figures 19(c) and (d) the first and last step respectively cross over the CoM. In these cases the M-L ground forces change from lateral to medial. Notice as well that each of these four cases begin in Quadrant IV, indicative of the CoP moving medially under the CoM.

Whenever a subject took a wide step, he or she tended to push off with the trailing foot. At $t=1 \mathrm{~s}$ in Figure 19(d), the participant changes from right (originating in QI) to left feet. This wide step causes the trailing foot to push off laterally, reaching (negative) $50 \mathrm{~N}$, a large increase over the course of the footstep. The same behaviour is seen at $t=1.5 \mathrm{~s}$ in Figure 19(e) and $t=1.25 \mathrm{~s}$ in Figure 19(f), where the wide step causes a lateral push-off much greater than the force over the rest of the step. Finally in Figure $19(\mathrm{~g})$, the push-off forces are seen in consecutive wide steps. Rönnquist [13] suggested that the M-L ground force was correlated to bridge phase; the results here support and clarify those of Rönnquist while also attributing the changes in ground force to the step width and CoP-CoM separation.

The figures also show that the offset in the M-L ground force from step to step is generally proportional to the width of the step. The participant in Figure 19(c) slightly crosses her second step over her first. As a result, no obvious offset occurs in the ground force, which incidentally is quasi-sinusoidal in phase with the structure. Figure 19(b) depicts a trial with a small and larger offset. The subject takes a small second step; even though a large transient peak in the ground force is evident, the steady state of the rest of the footstep is less than $30 \mathrm{~N}$ greater than the force at the end of the previous step. The width of the third step is approximately $160 \mathrm{~mm}$ to the right of the second step. This large step is met with an approximately $90 \mathrm{~N}$ offset in the M-L force. Finally, the steps in Figure 19(e) are among the largest at about 160 and $170 \mathrm{~mm}$. They correspond to force offsets of 70 and $90 \mathrm{~N}$ respectively. If the participants were walking at approximately the same speed the correlations between bridge phase and ISW can be extended to a relationship between phase and M-L ground force.

Most of the figures shown exhibit the flattening of the CoM trajectory indicative of a U-shaped, fixedin-space strategy. Traditional IPM models suggest that CoM trajectory should be near sinusoidal [19, 23], but the CoM in Figures 19(a) to (c) and (f) all exhibit three linear periods separated by small transitions. These flat regions occur when the CoM is moving vertically, as seen in the accompanying frontal plane trajectories. Figure 19(h) is unique in that the CoM exhibits a large range in the first step, followed by almost zero lateral translation in the second step, followed by a large range again in the third.

\footnotetext{
${ }^{3}$ Claff [23] investigates an updated model called the Modified Inverted Pendulum using the real CoM-CoP separation in his doctoral thesis.
} 


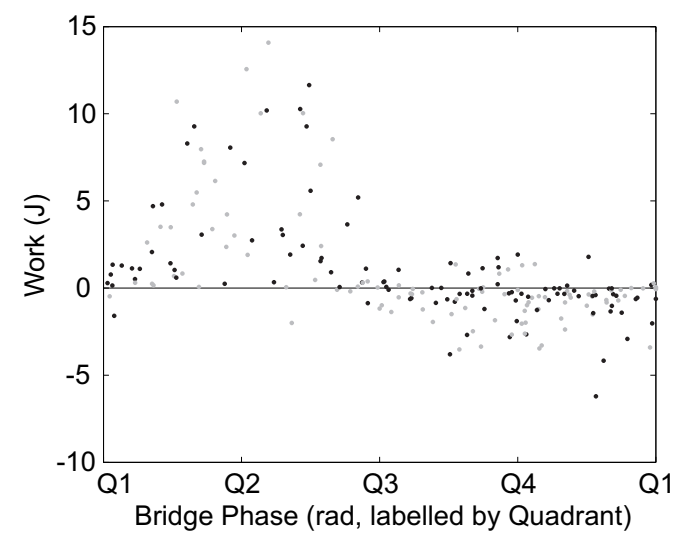

Figure 20: Energy contributed to or absorbed from the bridge in the form of work. Work done by left (gray) and right (black) footsteps versus phase of bridge at heel-strike

\subsubsection{Fitting the Inverted Pendulum Model}

One of the major deficiencies of the Inverted Pendulum Model on stable ground is that the model does not capture the higher frequency content of real M-L ground forces [23]. The plots of Figure 19 show a wide variety of acute oscillations in the forces recorded by the plates. Here as well, the model fails to account for these high-frequency oscillations. Ultimately, neither the CoP nor CoM exhibited the high-frequency content necessary to predict the frequency content of the ground forces, so these models are too simple to obtain a perfect match. This is comparable to the result found by Carroll et al. [15].

Nonetheless the amplitude of the models and the overall fit is good. In most cases the IPM matches the amplitude of the ground force well. In particular, the IPM usually reflects the offsets from step to step and matches the quasi-sinusoidal force pattern of the QIII and QIV samples.

In spite of the deficiencies, the IPM not only reproduced the general amplitudes of the M-L ground forces, but did so regardless of foot placement. Whether the steps were wide or crossed, the model was reasonably close to correct. This shows that even with deficiencies, the motion of a human on a moving surface is similar to an inverted pendulum with base motion.

\subsection{Energy Contributions to the Swaying Bridge}

One mechanical aspect of the bridge-pedestrian system that may be useful for engineers is the work conducted by a pedestrian on the swaying bridge. Work is the integral of power $(P)$ over time,

$$
W=\int P \mathrm{~d} t=\int F_{x} v_{x} \mathrm{~d} t .
$$

The power input to the bridge is equivalent to the M-L ground force $\left(F_{x}\right)$ of a footstep times the lateral velocity $\left(v_{x}\right)$ of the bridge at each time step. The power is then integrated over the duration of the footstep using the trapezoidal rule.

Figure 20 shows the total work done by each footstep as a function of the quadrant of heel-strike. Positive work represents energy added to the bridge while negative work is energy removed. The maximum work is done by heel-strikes early in QII and the maximum negative work by heel-strikes in mid-QIII and mid-QIV. Notice as well that there are some instances of positive work by heel-strikes in QIV.

Since the ground force and bridge velocity evolve over the duration of a footstep, Figure 21 shows a simple schematic of how the total work done by a footstep is dependent upon the quadrant in which heelstrike occurs. Heel-strikes in QI and QII have high positive work because most of the footstep duration 


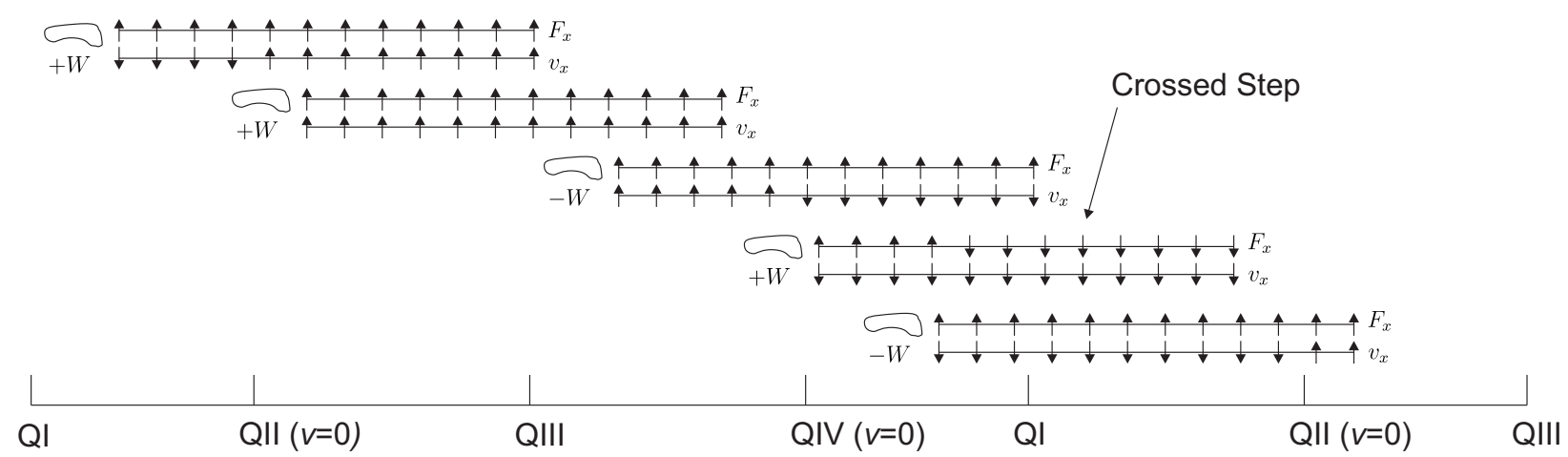

Figure 21: Schematic diagram indicating the relative contribution of lateral force and bridge velocity to the sign of total work done by a left footstep. Each bar represents the duration of a footstep, expressed in terms of bridge quadrants.

occurs when velocity is in the same direction as the force. Thus a heel-strike at the start of QII will exhibit maximum positve work since there is no period where velocity opposes force.

For heel-strikes occurring in QIII and QIV, much of the footstep duration is spent with velocity opposing force. Given Equation 6, the work done on the bridge is thus negative because the ground force and the bridge velocity are opposite signs. The caveat to this rule, however, is that if a footstep becomes crossed, the CoM-CoP separation switches sign, so the force also switches sign. $F_{x}$ and $v_{x}$ are then in the same direction, and positive work is developed. Since the magnitude of a crossed step is most likely to be greatest for a heel-strike at the start of QIV, this is the time when the local positive maximum work occurs.

Steps taken in QI and QII almost always contribute energy to the bridge due to the CoP diverging from the CoM. During QIII and QIV, in contrast, the work is typically negative; energy is absorbed from the bridge as the CoM and CoP converge.

These data correspond well to those of Bocian et al. [31], who similarly investigated the work done by a series of footsteps, but on a subject-by-subject basis. Their study depicted the progression of work done over at least a minute by an individual on an oscillating treadmill. They found that the total work done by consecutive footsteps progressed through peaks and troughs, with positive amplitude on the range of three to five factors greater than the negative work amplitude. The Oxford study corroborates this finding. The QII positive maximum (14 J) appears to be multiple times higher than the QIII and QIV negative maxima (4 and $6 \mathrm{~J})$. This is likely related to ISW. The wider ISW during QII steps makes it easier to push off, increasing the positive work done on the bridge, whereas the narrow ISW during QIV steps promotes a narrow footstep, or a M-L force near zero.

In the same study, Bocian et al. [31] show that the work of a footstep is much more likely to be positive than negative. The Oxford data does not support this assessment (47.6\% positive-work footsteps), but in principle, it should. The fact that QI and QII heel-strikes almost exclusively yield positive work whilst QIII and QIV heel-strikes can be positive or negative would make it logical - assuming an even distribution of heel-strikes - for a higher ratio of positive-work footsteps to be present. The Oxford tests incurred 56.7\% heel-strikes in Quadrants III and IV, which may have skewed the relative quantity of positive versus negative work samples.

That said, an important qualifier should be made. The work results presented in this study are based on subjects whose gait cycle frequency $(0.94 \mathrm{~Hz})$ is slightly greater than the bridge sway frequency $(0.67 \mathrm{~Hz})$; a footstep occurs in less than half of a bridge cycle. For different frequency ratios, the shape of Figure 20 is likely to vary since the footstep duration would encompass a higher or lower proportion of the bridge cycle.

The three graphs in Figure 22 depict three three-step plots. The first two depict walking that is nearly in 

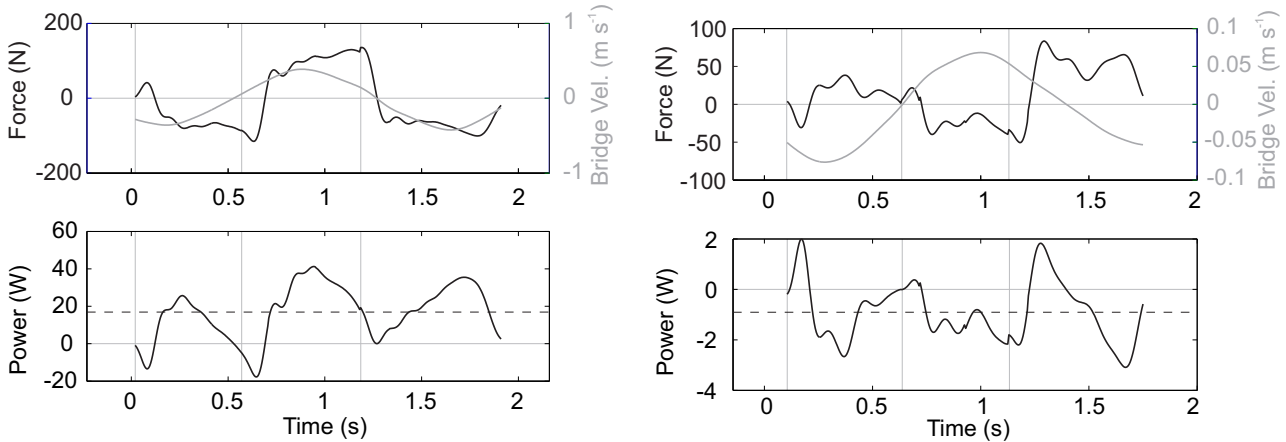

(a)

(b)
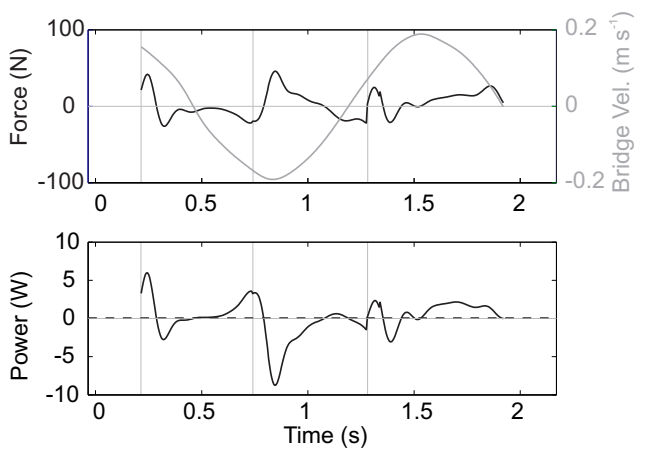

(c)

Figure 22: Force (top, black) and power (bottom) contribution of three trials compared to bridge velocity (top, gray). Mean threestep power (bottom, dash). Grid lines indicate heel-strike times. Sign and magnitude of the mean power represents (a) high energy contributed to the bridge, (b) high energy removed from the bridge, and (c) zero net energy contribution.

phase (successive QII steps) and in anti-phase (successive QIV steps) with the bridge's swinging, causing the mean power (ergo energy contribution) to be positive and negative respectively. Figure (c) depicts footsteps that are crossed, with a mean power of approximately zero. These plots show how consecutive steps contribute to a net positive or negative level of work done depending on the quadrant of heel-strike.

The assessment of work done on the bridge has many implications. The propagation of bridge vibration - which is periodic - does not have to be from periodic forces. Regardless of the frequency content of an individual's ground forces, as long as the net work on the bridge over a series of successive steps is positive, vibration will propagate. That said, locking-in with the structure will rapidly propagate bridge vibrations if the locked-in steps are in phase with the structure. The second implication is that since the maximum amount of negative work an individual can exert during a single 'normal' step appears to be much less than the amount of positive work, the propagation of structural vibrations is more likely than its attenuation (assuming $v_{x}$ is not zero). Third, a pedestrian can attenuate structural vibrations by locking-in in anti-phase with the structure, which imparts negative work over consecutive steps. Of course, this only works so long as the pedestrian avoids taking crossover steps, perhaps requiring the adoption of a fixed-to-base strategy to ensure the CoM remains on the 'correct' side of the CoP.

Another means of attenuating vibrations is to stop walking so that the lateral force is zero. The attenuation will be slower than by doing negative work on the bridge, but the strategy might be easier for the poor pedestrian than trying to absorb the energy of the bridge! 
The energy transfer between pedestrians and the structure can be thought of as a form of damping - with energy added to the structure implying negative damping, and energy removed implying positive damping. Bocian et al. [28] developed a method of estimating the effect of walkers on the bridge's oscillation frequency, and proposed a critical level of structural damping required to maintain stability in the presence of walkers. Their method was based on a biomechanically-inspired mathematical model of walking forces, but has been applied here using measured force data.

In the Oxford tests the bare bridge's natural frequency was $0.67 \mathrm{~Hz}$ and the mass ratio $(\mu)$ due to walkers varied between 4.5 and $9.5 \%$. The effect of walkers on the bridge's oscillation frequency was found to be negligible - this is broadly consistent with Fig 7(a) in the paper by Bocian et al. [28], which indicates a frequency ratio close to unity for these parameter values.

Turning to the damping, Bocian et al. [28] define a stability boundary as a function of bridge frequency and the pedestrian to structure mass ratio. If the unloaded damping ratio of a structure is below its stability boundary, instability could occur. In the Oxford tests, the structural damping was very low (below 0.001), in order to elicit structural instability from the participants' walking, which was frequently observed. Using Equations 9-12 and 17-19 from Bocian et al. [28], stability boundaries were determined using the Oxford walking data. The boundaries ranged from -0.07 to 0.10 with a mean of 0.0026 . Since the damping ratio of the Oxford bridge was nearly zero, any test producing a positive stability limit likely exhibited lateral instability because the bridge's damping ratio was below the calculated limit. Conversely, any test producing a negative stability limit indicated that the structural damping ratio did not matter; instability theoretically would not occur.

With a frequency of $0.67 \mathrm{~Hz}$ and $\mu=10 \%$, Fig 7(b) by Bocian et al. [28] suggests a stability boundary at a damping ratio of approximately 0.06 . This is broadly consistent with the Oxford test results. While the worst-case test gave a value of 0.10 , most of the positive Oxford boundary limits were between zero and 0.06. These figures therefore suggest a good level of agreement between the Oxford experiments and the model by Bocian et al. [28] given the range mass ratios tested in the experiments.

\section{Conclusions}

These tests and the presented results offer significant advancements from many previous studies. First, motion of the structure was entirely in response to forces imparted by the test subjects rather than being artificially actuated. Second, the subjects could freely select and alter their walking speed in a natural environment. Third, the combination of deck force plates, insole pressure sensors, and motion capture yielded very rich datasets. These improvements were beneficial because they allowed for the replication and analysis of a true human-structure feedback system, such as might be felt on a real bridge.

Three major observations were made pertaining to foot placement and Centre of Pressure motion. First, the instantaneous step width was found to be correlated to the the phase of the bridge at the previous heelstrike. Second, the phase of the bridge at heel-strike was a major determinant of CoP displacement over the course of the subsequent stance phase, particularly for higher structural amplitudes. Third, the net step width was minimally correlated to bridge motion, suggesting that a person will usually step in a 'normal' position as if walking on stable ground. These three observations suggest that foot and CoP position can be predicted for any given bridge phase. If the heel-strike timings are also known (or assumed) then the foot placements for a whole bridge crossing can be predicted.

An observation linking ISW to ground force confirmed previous studies: ISW was found to be proportional to the ground force offset from step to step. Since the CoM-CoP separation is also proportional to the step width, the possibility arises for the future development of a model that describes M-L ground force solely in terms of structural motion and a few anatomic measurements. 
Three observations were made regarding Centre of Mass motion. First, the pedestrians generally fixed their CoM to the global reference frame, allowing their feet to move freely beneath them. Second, CoM trajectories in the frontal plane varied greatly, ranging from figure eight shape to U-shaped. Still, some patterns emerged for different bridge-to-pedestrian relationships. Third, while the trajectories need further analysis, the IPM is a reasonably good predictor of ground forces on a moving base.

Finally, it was observed that pedestrian work on the structure was highly dependent on the bridge phase at heel-strike. Development of positive or negative work was strongly yet unequally correlated to diverging or converging $\mathrm{CoP}$ trajectories, inviting vibration propagation if the system damping ratio is low enough. In addition, the data were used to corroborate the stability boundaries produced by Bocian et al. [28], which predict when lateral instability is likely to occur.

Here it has been shown that structural position and velocity are integral for the development of NSW, ISW, CoP trajectory, CoM trajectory, CoM-CoP separation, M-L ground force development, and total work. Existing models generally do a reasonable job of predicting the overall character of ground forces, but future research should further incorporate structural motion into kinematic and kinetic models of gait.

\section{Acknowledgements}

We would like to thank Oxford University Press for their financial support via the Oxford University Clarendon Fund. The contributions of Clive Baker, Alice Kaye, Andrew Mather, and Grace Selley to the design and construction of the bridge are also gratefully acknowledged. Finally, the testing would not have been possible without all the volunteer walkers and the generous assistance of Brian Shiel.

\section{References}

[1] P. Dziuba, G. Grillaud, O. Flamand, S. Sanquier, Y. Tetard, La passerelle Solférino comportement dynamique (dynamic behaviour of the Solferino bridge), Bull Ouvrages Metalliques 1 (2001) 34-57, [In French].

[2] P. Dallard, A.J. Fitzpatrick, A. Flint, S. L. Bourva, A. Low, R.M. Ridsdill Smith, M. Willford, The London Millennium Footbridge, The Structural Engineer 79 (22) (2001) 17-33.

[3] T. Morbiato, R. Vitaliani, A. Saetta, Numerical analysis of a synchronization phenomenon: Pedestrian-structure interaction, Computers and Structures 89 (17-18) (2011) 1649-1663.

[4] H. Bachmann, W. Ammann, Vibrations in Structures: Induced by Man and Machines, International Association for Bridge and Structural Engineering, Zürich, third edn., 1987.

[5] G. Giakas, V. Baltzopoulos, Time and frequency domain analysis of ground reaction forces during walking: an investigation of variability and symmetry, Gait \& Posture 5 (3) (1997) 189-197.

[6] M. Bocian, J.H.G. Macdonald, J.F. Burn, Probabilistic criteria for lateral dynamic stability of bridges under crowd loading, Computers and Structures 136 (2014) 108-119.

[7] E.T. Ingólfsson, C.T. Georgakis, A stochastic load model for pedestrian-induced lateral forces on footbridges, Engineering Structures 33 (12) (2011) 3454-3470.

[8] V. Racic, J.M.W. Brownjohn, Stochastic model of near-periodic vertical loads due to humans walking, Advanced Engineering Informatics 25 (2) (2011) 259-275.

[9] F. Ricciardelli, A.D. Pizzimenti, Lateral walking-induced forces on footbridges, Journal of Bridge Engineering 330 (6) (2007) $1265-1284$

[10] E.T. Ingólfsson, C.T. Georgakis, F. Ricciardelli, J. Jönsson, Experimental identification of pedestrian-induced lateral forces on footbridges, Journal of Sound \& Vibration 330 (6) (2011) 1265-1284.

[11] P. Dallard, T. Fitzpatrick, A. Flint, A. Low, R.R. Smith, M. Willford, M. Roche, London Millennium Footbridge: PedestrianInduced Lateral Vibration, Journal of Bridge Engineering 6 (6) (2001) 412-417.

[12] A. McRobie, G. Morgenthal, J. Lasenby, M. Ringer, Section model tests on human-structure lock-in, Proceedings of the Institution of Civil Engineers: Bridge Engineering 156 (2) (2003) 71-79.

[13] A. Rönnquist, Pedestrian Induced Lateral Vibrations of Slender Footbridges, Ph.D. thesis, Norwegian University of Science and Technology, 2005. 
[14] F.A. McRobie, Long-term solutions of Macdonald's model for pedestrian-induced lateral forces, Journal of Sound \& Vibration 332 (11) (2013) 2846-2855.

[15] S.P. Carroll, J.S. Owen, M.F.M. Hussein, Experimental identification of the lateral human-structure interaction mechanism and assessment of the inverted-pendulum biomechanical model, J Sound Vib 333 (22) (2014) 5865-5884.

[16] M. Bocian, J.H.G. Macdonald, J.F. Burn, D. Redmill, Experimental identification of the behaviour of and lateral forces from freely-walking pedestrians on laterally oscillating structures in a virtual reality environment, Engineering Structures 105 (2015) 62-76.

[17] C.D. MacKinnon, D.A. Winter, Control of Whole Body Balance in the Frontal Plane During Human Walking, Journal of Biomechanics 26 (6) (1993) 633-644.

[18] A.L. Hof, M.G.J. Gazendam, W.E. Sinke, The condition for dynamic stability, Journal of Biomechanics 38 (1) (2005) 1-8.

[19] J.H.G. Macdonald, Lateral excitation of bridges by balancing pedestrians, Proceedings of the Royal Society A: Mathematical, Physical, and Engineering Sciences 465 (2104) (2009) 1055-1073.

[20] D. Claff, M.S. Williams, A. Blakeborough, J. Stebbins, Medial-Lateral Gait Patterns of Healthy Adult Walkers, in: Conference Proceedings of the Society for Experimental Mechanics Series, vol. 39, 337-348, 2013.

[21] J.H.G. Macdonald, Pedestrian-induced vibrations of the Clifton Suspension Bridge, UK, Proceedings of the Institution of Civil Engineers: Bridge Engineering 161 (2) (2008) 69-77.

[22] D. Claff, M.S. Williams, A. Blakeborough, Integrating Force Plates into a Laterally Oscillating Footbridge, in: Footbridges: Past, present, and future, London, 174-175, proceedings of Footbridge 2014: 5th International Conference, 2014.

[23] D. Claff, Pedestrian-Induced Lateral Excitation of Footbridges, Ph.D. thesis, University of Oxford, 2016.

[24] Oxford Foot Model, Oxford Foot Model, https://www.vicon.com/products/software/oxford-foot-model, online; accessed 2017-04-08, 2015.

[25] R.A. Brady, B.T. Peters, J.J. Bloomberg, Strategies of healthy adults walking on a laterally oscillating treadmill, Gait \& Posture 29 (4) (2009) 645-649.

[26] Y. Fujino, B.M. Pacheco, S.-I. Nakamura, P. Wamitchai, Synchronization of human walking observed during lateral vibration of a congested pedestrian bridge, Earthquake Engineering \& Structural Dynamics 22 (9) (1993) 741-758.

[27] L. Tesio, V. Rota, C. Chessa, L. Perucca, The 3D path of body centre of mass during adult human walking on force treadmill, Journal of Biomechanics 43 (5) (2010) 938-944.

[28] M. Bocian, J.H.G. Macdonald, J.F. Burn, Biomechanically inspired modelling of pedestrian-induced forces on laterally oscillating structures, Journal of Sound \& Vibration 331 (16) (2012) 3914-3929.

[29] A.L. Hof, The 'extrapolated center of mass' concept suggests a simple control of balance in walking, Human Movement Science 27 (1) (2008) 112-125.

[30] L.I.E. Oddsson, C. Wall III, M.D. McPartland, D.E. Krebs, C.A. Tucker, Recovery from perturbations during paced walking, Gait \& Posture 19 (1) (2004) 24-34.

[31] M. Bocian, J.F. Burn, J.H.G. Macdonald, J.M.W. Brownjohn, From phase drift to synchronisation - pedestrian stepping behaviour on laterally oscillating structures and consequences for dynamic stability, Journal of Sound \& Vibration 392 (2017) 382-399. 